\title{
A Polychromatic Ramsey Theory for Ordinals
}

\author{
Martin Huschenbett ${ }^{1}$ and Jiamou Liu ${ }^{2}$ \\ 1 Institut für Theoretische Informatik \\ Technische Universität Ilmenau, Germany \\ martin.huschenbett@tu-ilmenau.de \\ 2 School of Computing and Mathematical Sciences \\ Auckland University of Technology, New Zealand \\ jiamou.liu@aut.ac.nz
}

\begin{abstract}
The Ramsey degree of an ordinal $\alpha$ is the least number $n$ such that any colouring of the edges of the complete graph on $\alpha$ using finitely many colours contains an $n$-chromatic clique of order type $\alpha$. The Ramsey degree exists for any ordinal $\alpha<\omega^{\omega}$. We provide an explicit expression for computing the Ramsey degree given $\alpha$. We further establish a version of this result for automatic structures. In this version the ordinal and the colouring are presentable by finite automata and the clique is additionally required to be regular. The corresponding automatic Ramsey degree turns out to be greater than the set theoretic Ramsey degree. Finally, we demonstrate that a version for computable structures fails.
\end{abstract}

\section{Introduction}

The (countably) infinite Ramsey's theorem states that any edge colouring of a countably infinite complete graph admits a complete monochromatic infinite clique. If we arrange the nodes in this graph into a well-ordering of order type $\omega$, Ramsey's theorem guarantees the existence of a subordering of order type $\omega$ such that all pairs of its elements have the same colour. More specifically, by a standard partition of a set $A$ we mean a partition of all 2-element subsets (or edges) of $A$ into a finite number $k$ of classes, where $k \geq 1$. A homogeneous set with respect to a standard partition of $A$ is a subset $B \subseteq A$ such that all edges of $B$ belong to one class of the partition. If $\alpha$ and $\beta$ are ordinals, one writes $\alpha \rightarrow(\beta)$ for the fact that whenever $(A, \leq)$ has order type $\alpha$, any standard partition of $A$ admits a homogeneous subset $B$ such that the suborder $(B, \leq \uparrow B)$ has order type $\beta$. Ramsey's theorem can thus be stated as $\omega \rightarrow(\omega)$.

A question then arises as to whether one can extend the above statement to larger ordinals. Erdős and Rado gave a negative answer to this question for countable ordinals: For any countable well-ordering $L$ there is a partition of edges of $L$ such that any infinite homogeneous subset of $L$ has order type $\omega$ [6]. Hence for any countable ordinal $\alpha, \alpha \nrightarrow \rightarrow(\omega+1)$. This result is the start of a vast amount of works on the partition relations of ordinals, which has become a central notion of combinatorial set theory [5]. 
Since the 1970s, there has been another well-established extension to the Ramsey's theorem. The goal is to investigate the effective content of the homogeneous sets in computable standard partitions. Recall that a structure is computable if its domain as well as its atomic functions and predicates are decidable by Turing machines. Specker showed that the original statement in Ramsey's theorem cannot be made effective: there exists a computable standard partition of a computable copy of $\omega$ such that no infinite homogeneous set is computable [17]. Jockusch then showed that the infinite homogeneous sets of standard partitions of $\omega$ do not even necessarily belong to $\Sigma_{2}$. On the other hand, infinite homogeneous sets are guaranteed to exist in $\Pi_{2}$ [8].

More recently, attention has been given to automatic structures. These are structures that are defined in a similar way as computable structures except the "Turing machines" in the definition is replaced by "finite automata". Hence automatic structures form a subclass of computable structures. A main line of research in the study of automatic structures is to understand automaticity in classical theorems. Here, as opposed to computable structures for which numerous classical results (such as König's lemma and Ramsey's theorem) fail in the computable case, the automatic counterparts of these theorems hold. For example, Rubin proved that in any automatic standard partition of an infinite regular language there exists necessarily homogeneous sets that are recognisable by finite automata [15]. This result suggests that it makes sense to build a Ramsey theory on automatic ordinals.

We mention here that a standard partition can be viewed as a colouring function that maps the set of edges to a finite number of colours. The homogeneous sets mentioned above are thus "monochromatic". In this paper we consider colourings that consist of more than two colours and " $\ell$-chromatic" subsets for some bounded number $\ell$, that is, subsets whose edges are coloured by no more than $\ell$ colours. It is natural to ask the following: Let $\alpha$ be an ordinal.

1. Is there a number $\ell \in \mathbb{N}$ such that any edge colouring of $\alpha$ contains an $\ell$-chromatic subset of order type $\alpha$ ?

2. If such a number $\ell$ in the above question exists, how large must it be?

We call the least number $\ell$ that satisfies the first question the Ramsey degree of the ordinal $\alpha$. Williams in [19, Thm. 7.2.7] showed that the Ramsey degree exists for any ordinal $\omega^{n}$ where $n \in \mathbb{N}$. Here we further provide a formula for computing the Ramsey degree of an arbitrary $\alpha<\omega^{\omega}$; see Theorem 3.3 and Appendix A.

We then explore the same questions as above restricting to copies of ordinals and colourings that are finite-automata presentable, and regular $\ell$-chromatic sets. For any ordinal $\alpha<\omega^{\omega}$, we show that the corresponding automatic Ramsey degree exists for $\alpha$ and give an explicit expression for computing it. The automatic Ramsey degree of $\alpha$ turns out to be strictly greater than its Ramsey degree if $\omega^{2} \leq \alpha<\omega^{\omega}$. A by-product of our investigation is a similar result on automatic complete bipartite graphs, where each bipartition is an ordinal. Finally we briefly present a negative answer to the computable version of the 
above questions: For any $k \geq 1$, there is a computable edge colouring of the natural numbers using $k+1$ colours that does not admit any infinite $k$-chromatic computably enumerable subsets.

Related works. The notion of Ramsey degrees used here has appeared in different forms in the literature. The paper [18] contains several results discussing similar notions. The result that motivated our study is F. Galvin's unpublished theorem on rationals: For any edge colouring of $\eta$, the order type of rationals, there must be a 2-chromatic sub-copy of $\eta$ [7]. Pouzet and Sauer obtained a very similar result on the random graphs [12]. See [11] for an introduction on the automatic version of Ramsey's theorem.

Paper organisation. Section 2 introduces necessary background in Ramsey theory and automatic structure. Section 3 and Section 4 discusses Ramsey degrees in the general case and the automatic case respectively. Section 5 presents the computable case. Finally Section 6 discusses open problems.

\section{Preliminaries}

Throughout the whole paper, $\mathbb{N}$ denotes the natural numbers $1,2,3, \ldots$ and $\mathbb{N}_{0}$ denotes $\mathbb{N} \cup\{0\}$. We use the interval notation $[i, j]$ for the set $\{i, i+1, \ldots, j\}$.

Well-orderings and ordinals. A well-ordering is a linear ordering $\left(V ; \leq_{V}\right)$ with no infinite descending chains. For details on basic notions and results on wellorderings and ordinals the reader is referred to [14]. We view sets also as wellordered sets, i.e., a set $V$ also denotes a well-ordering $\left(V, \leq_{V}\right)$. By " $V$ has order type $\alpha$ " we mean " $\left(V ; \leq_{V}\right)$ has order type $\alpha$ ". By $U+V$ we mean the sum of the well-orderings $\left(U ; \leq_{U}\right)+\left(V ; \leq_{V}\right)$. If $U \subseteq V$ then we assume the ordering on $U$ is the same as the ordering on $V$ restricted to $U$.

Let $n \geq 0$. We view $\mathbb{N}^{n}$ as a well-ordered set using the order defined by $\left(x_{0}, \ldots, x_{n-1}\right)<\mathbb{N}^{n}\left(y_{0}, \ldots, y_{n-1}\right)$ if there is an $i \in[0, n-1]$ with $x_{i} \neq y_{i}$ and the least such $i$ satisfies $x_{i}<y_{i}$. Then $\mathbb{N}^{n}$ has order type $\omega^{n}$ and is regarded as the canonical representation of $\omega^{n}$. As we consider no other orders on $\mathbb{N}^{n}$ besides $\leq_{\mathbb{N}^{n}}$, we usually omit the subscript $\mathbb{N}^{n}$ from $\leq_{\mathbb{N}^{n}}$.

It is well-known that any ordinal $\alpha<\omega^{\omega}$ can be uniquely written in its Cantor normal form $\alpha=\omega^{n_{1}}+\omega^{n_{2}}+\cdots+\omega^{n_{r}}$ with $r \geq 0$ and $n_{1} \geq n_{2} \geq \cdots \geq n_{r} \geq 0$.

Finite automata and semigroups. We assume some familiarity with the basic concepts of (algebraic) automata theory (cf. [4]). Let $\Sigma$ be an alphabet. (Nondeterministic) finite automata (over $\Sigma$ ) and their languages are defined as usual.

A semigroup is a set $S$ equipped with an associative binary multiplication. Examples include the set $\Sigma^{*}$ with concatenation and the direct product of finitely many semigroups. A (semigroup) morphism is a map between two semigroups which preserves multiplication. The Myhill-Nerode theorem states that a language $L \subseteq \Sigma^{*}$ is regular if, and only if, there is a morphism $\eta: \Sigma^{*} \rightarrow S$ into a finite semigroup $S$ which recognises $L$, i.e., $L=\eta^{-1}(T)$ for some $T \subseteq S$. This 
theorem is effective in both directions, i.e., one can compute a morphism recognising $L$ from a finite automaton recognising $L$ and vice versa. For any finite number $L_{1}, \ldots, L_{n} \subseteq \Sigma^{*}$ of regular languages there exists a morphism into a finite semigroup which simultaneously recognises all the $L_{i}$.

An element $s \in S$ of a semigroup $S$ is idempotent if $s^{2}=s$. An idempotency exponent of $S$ is a number $K \geq 1$ such that $s^{K}$ is idempotent for all $s \in S$. Whenever $S$ is finite, any multiple of $|S|$ ! is an idempotency exponent of $S$.

Automatic structures. To recognise $n$-ary relations on $\Sigma^{*}$, we use finite automata which synchronously process $n$ input tapes in parallel. Formally, let $\diamond \notin \Sigma$ be an additional padding symbol and $\Sigma_{\diamond}=\Sigma \cup\{\diamond\}$. The convolution of a tuple $\bar{u}=$ $\left(u_{0}, \ldots, u_{n-1}\right) \in\left(\Sigma^{*}\right)^{n}$ is the word $\otimes \bar{u} \in\left(\Sigma_{\diamond}^{n}\right)^{*}$ of length $\max \left\{\left|u_{0}\right|, \ldots,\left|u_{n-1}\right|\right\}$ whose $k^{\text {th }}$ symbol is $\left(\sigma_{0}, \ldots, \sigma_{n-1}\right)$, where $\sigma_{i}$ is the $k^{\text {th }}$ symbol of $u_{i}$ if $k \leq\left|u_{i}\right|$, and $\diamond$ otherwise. A relation $R \subseteq\left(\Sigma^{*}\right)^{n}$ is automatic if the set $\otimes R=\{\otimes \bar{u} \mid \bar{u} \in R\}$ is a regular subset of $\left(\Sigma_{\diamond}^{n}\right)^{*}$.

A relational structure $\mathcal{A}=\left(A ; R_{1}, \ldots, R_{k}\right)$ consists of a set $A$, its domain, and relations $R_{1}, \ldots, R_{k}$ on $A$. A structure $\mathcal{A}$ is automatic if $A$ is a regular language (over some alphabet $\Sigma$ ) and the relations $R_{i}$ are automatic. In this situation, an automatic presentation of $\mathcal{A}$ is a tuple of finite automata recognising $A$ and the $\otimes R_{i}$, respectively. We denote by AUT the class of all automatic structures, which actually includes all regular languages. The main motivation for investigating automatic structures is the decidability of their first-order theories (cf. [1,9]).

Theorem 2.1 (Khoussainov, Nerode [9]). Every first-order definable relation $R$ on an automatic structure $\mathcal{A}$ is automatic and one can compute a finite automaton recognising $R$ from an automatic presentation of $\mathcal{A}$ and a first-order formula defining $R$. In particular, the first-order theory of $\mathcal{A}$ is decidable.

A well-ordering $A$ is automatic in the sense above if $A$ is a regular language and $\leq_{A}$ an automatic relation. Automatic well-orderings are a well studied subject.

Theorem 2.2 (Delhommé [3]). There is an automatic well-ordering of type $\alpha$ if, and only if, $\alpha<\omega^{\omega}$.

Theorem 2.3 (Khoussainov et. al [10]). Given an automatic presentation of a well-ordering, one can compute the Cantor normal form of its order type.

\section{Ramsey Relations and Ramsey Degrees}

\subsection{Ordinal Ramsey Relation}

We use $[V]^{2}$ to denote the set of all 2-element subsets of a set $V$. For convenience we view $[V]^{2}$ as the irreflexive and symmetrical relation $\left\{(x, y) \in V^{2} \mid x \neq y\right\}$. It is customary to view standard partitions as colourings, which is the notion we adopt in this paper. Let $\alpha$ be an ordinal. An $\alpha$-colouring is a function $C:[V]^{2} \rightarrow Q$ where $V$ is a set of order type $\alpha$ and $Q$ is a finite set of colours. When $\alpha$ is clear from context we simply call $C$ a colouring (on $V$ ). Let $X \subseteq V$. We use $C(X)$ to 
denote the set $\left\{C(A) \mid A \in[X]^{2}\right\}$. Let $D \subseteq Q$. The set $X$ is $D$-chromatic w.r.t. $C$ if $C(X) \subseteq D$. In this case, we also say that $X$ is $|D|$-chromatic.

Let $\alpha, \beta$ be two ordinals, $k \in \mathbb{N}$, and $\ell \in \mathbb{N}_{0}$. The ordinal Ramsey relation is written as $\alpha \rightarrow(\beta)_{k, \ell}$ and denotes the fact that any $\alpha$-colouring $C:[V]^{2} \rightarrow[1, k]$ admits an $\ell$-chromatic subset $X \subseteq V$ of order type $\beta$. We are interested in the Ramsey degrees of ordinals, which is defined below.

Definition 3.1. Let $\alpha<\omega^{\omega}$ be an ordinal. The least $\ell \in \mathbb{N}_{0}$ such that $\alpha \rightarrow(\alpha)_{k, \ell}$ for all $k \in \mathbb{N}$ is called the Ramsey degree of $\alpha$ and denoted by $d_{R}(\alpha)$.

The countably infinite case of Ramsey's theorem states that $d_{R}(\omega)=1$ [13]. Williams in his book [19, Thm. 7.2.7] proved the following result, which extends Ramsey's theorem to ordinals $\omega^{n}$ where $n \in \mathbb{N}$.

Theorem 3.2 ([19, Thm. 7.2.7]). For any $n \in \mathbb{N}$ there is an $\ell \in \mathbb{N}_{0}$ such that

$$
\forall k \in \mathbb{N}: \omega^{n} \rightarrow\left(\omega^{n}\right)_{k, \ell} .
$$

The proof of Thm. 3.2 from [19] does not provide us the value of $d_{R}\left(\omega^{n}\right)$. We provide a proof for the following theorem in Appendix A.

Theorem 3.3. For all ordinals $\alpha<\omega^{\omega}$, we have

$$
d_{R}(\alpha)=\sum_{1 \leq i \leq r} \sum_{1 \leq j \leq n_{i}}\left(\begin{array}{c}
2 j-1 \\
j
\end{array}\right)+\sum_{1 \leq i<j \leq r}\left(\begin{array}{c}
n_{i}+n_{j} \\
n_{i}
\end{array}\right)
$$

where $\alpha=\omega^{n_{1}}+\cdots+\omega^{n_{r}}$ with $r \geq 0$ and $n_{1} \geq \cdots \geq n_{r} \geq 0$ is the Cantor normal form of $\alpha$.

Let $\mathcal{C}$ be a class of colourings and $\mathcal{D}$ a class of sets. We write

$$
(\alpha: \mathcal{C}) \rightarrow(\beta: \mathcal{D})_{k, \ell}
$$

if any $\alpha$-colouring $C:[V]^{2} \rightarrow[1, k]$ in $\mathcal{C}$ admits an $\ell$-chromatic set $X \subseteq V$ of order type $\beta$ such that $X \in \mathcal{D}$. We say that a colouring $C:[A]^{2} \rightarrow Q$ is automatic if the well-ordering $A$ is automatic and the relation $C^{-1}(q)$ is automatic for each $q \in Q$. The following is an automatic version of Ramsey's theorem.

Theorem 3.4 (Rubin [15]). Let $k \in \mathbb{N}$ be a number. We have

$$
(\omega: \text { AUT }) \rightarrow(\omega: \text { AUT })_{k, 1} .
$$

This theorem is effective in the following sense: Given an automatic presentation of an $\omega$-colouring on $A$, one can compute a finite automaton recognising a monochromatic, regular set $X \subseteq A$ of order type $\omega$. A main goal of the paper is to extend Thm. 3.4 by presenting an automatic version of Thm. 3.3. 


\subsection{Bipartite Ordinal Ramsey Relation}

As part of our investigation we also introduce a bipartite analogue of the Ramsey relation on ordinals. Let $\alpha$ and $\beta$ be ordinals. A bipartite $(\alpha, \beta)$-colouring is a function $C: U \times V \rightarrow Q$ where $U$ and $V$ have respectively order types $\alpha$ and $\beta$ and $Q$ is a finite set of colours. When $\alpha$ and $\beta$ are clear we simply call $C$ a bipartite colouring (on $(U, V)$ ).

Let $C: U \times V \rightarrow Q$ be a bipartite colouring. We write $(X, Y) \subseteq(U, V)$ to denote the fact that $X \subseteq U$ and $Y \subseteq V$. Let $\ell \in \mathbb{N}_{0}$. A pair $(X, Y) \subseteq(U, V)$ is $\ell$-chromatic w.r.t. $C$ if $|C(X \times Y)| \leq \ell$. We say that the pair $(X, Y)$ has order type $(\gamma, \delta)$ if $X$ and $Y$ have order type $\gamma$ and $\delta$, respectively.

Let $\alpha, \beta, \gamma, \delta$ be ordinals, $k \in \mathbb{N}$, and $\ell \in \mathbb{N}_{0}$. The bipartite ordinal Ramsey relation is written as $(\alpha, \beta) \rightarrow(\gamma, \delta)_{k, \ell}$ and denotes the fact that any $(\alpha, \beta)$ colouring $C: U \times V \rightarrow[1, k]$ admits an $\ell$-chromatic pair $(X, Y) \subseteq(U, V)$ of order type $(\gamma, \delta)$. The finite version of Ramsey theory on complete bipartite graphs has been well studied; see [2] for example. Here we study the bipartite ordinal Ramsey relation when the ordinals involved are $\omega^{n}$ where $n \in \mathbb{N}$. We define bipartite Ramsey degrees as follows.

Definition 3.5. Let $m, n \geq 0$. The least $\ell \in \mathbb{N}$ such that $\left(\omega^{m}, \omega^{n}\right) \rightarrow\left(\omega^{m}, \omega^{n}\right)_{k, \ell}$ for all $k \geq 1$ is called the Ramsey degree of $\left(\omega^{m}, \omega^{n}\right)$ and denoted by $d_{R}\left(\omega^{m}, \omega^{n}\right)$.

Appendix A contains also the proof of the following result.

Theorem 3.6. For all $m, n \in \mathbb{N}_{0}$, we have

$$
d_{R}\left(\omega^{m}, \omega^{n}\right)=\left(\begin{array}{c}
m+n \\
m
\end{array}\right) .
$$

In the following we generalise the above notion to specific classes of bipartite colourings. Let $\mathcal{C}$ be a class of colourings and $\mathcal{D}$ a class of sets. We write

$$
(\alpha, \beta: \mathcal{C}) \rightarrow(\gamma, \delta: \mathcal{D})_{k, \ell}
$$

for the fact that any $(\alpha, \beta)$-colouring $C: U \times V \rightarrow[1, k]$ in $\mathcal{C}$ admits an $\ell$-chromatic pair of sets $(X, Y) \subseteq(U, V)$ of order type $(\gamma, \delta)$ and $X, Y \in \mathcal{D}$.

\section{The Automatic Case}

In this section, we investigate an automatic analogue of the Ramsey degree from the previous section. The highlight is Thm. 4.7 which states that this degree exists for each ordinal $\alpha<\omega^{\omega}$ and provides a formula to compute its value. ${ }^{3}$

Definition 4.1. Let $\alpha<\omega^{\omega}$ be an ordinal. If there exists an $\ell \in \mathbb{N}_{0}$ such that $(\alpha: \mathrm{AUT}) \rightarrow(\alpha: \mathrm{AUT})_{k, \ell}$ for all $k \in \mathbb{N}$, the least such $\ell$ is called the automatic Ramsey degree of $\alpha$ and denoted by $d_{R, \mathrm{AUT}}(\alpha)$.

Similarly, we define the automatic (bipartite) Ramsey degree $d_{R, \mathrm{AUT}}(\alpha, \beta)$ for ordinals $\alpha, \beta<\omega^{\omega}$.

\footnotetext{
${ }^{3}$ Details missing from the proofs in this section can be found in Appendix B.
} 


\subsection{Automatic Well-Orderings of Type $\omega^{n}$}

Our main tool in the investigation of the automatic Ramsey degree is Thm. 4.2 below which roughly states that every automatic well-ordering of type $\omega^{n}$ contains a simple automatic subordering of the same order type.

We call a map $f: \mathbb{N}^{n} \rightarrow \Sigma^{*}$ is presentable if there are $u_{0}, \ldots, u_{n-1}, u_{n} \in \Sigma^{*}$ and $v_{0}, \ldots, v_{n-1} \in \Sigma^{+}$such that

$$
f\left(x_{0}, \ldots, x_{n-1}\right)=u_{0} v_{0}^{x_{0}-1} u_{1} v_{1}^{x_{1}-1} \cdots u_{n-1} v_{n-1}^{x_{n-1}-1} u_{n}
$$

for all $\bar{x} \in \mathbb{N}^{n}$. The tuple $\left(u_{0}, v_{0}, \ldots, u_{n-1}, v_{n-1}, u_{n}\right)$ is called presentation of $f$. If there exists a $K \geq 1$ such that $\left|u_{i}\right|=\left|v_{i}\right|=K$ for $0 \leq i<n$ and $\left|u_{n}\right| \leq K$, we say that $f$ is $(K$-) uniformly presentable and speak of a $(K$-)uniform presentation.

Theorem 4.2. Let $n \geq 0$. For every automatic well-ordering $A$ of type $\omega^{n}$ there exists a uniformly presentable embedding $f: \mathbb{N}^{n} \hookrightarrow A$.

Proof. Let $A \subseteq \Sigma^{*}$ be an automatic well-ordering of type $\omega^{n}$. We first show the existence of a (possibly non-uniformly) presentable embedding $f^{\prime}: \mathbb{N}^{n} \hookrightarrow A$ by induction on $n$. The claim is trivial for $n=0$. Therefore, we assume $n \geq 1$.

We define an equivalence relation $\sim$ on $A$ by $u \sim v$ if the interval $[u, v)$ has order type strictly below $\omega^{n-1}$. The $\sim$-class of any $u \in A$ is denoted by $[u]$. It is an interval of $A$ which has order type $\omega^{n-1}$. The set $P=\{\min [u] \mid u \in A\}$ is a system of representatives w.r.t. $\sim$ which has order type $\omega$. Thus, $A=\sum_{u \in P}[u]$ is the unique representation of $A$ as an $\omega$-sum of copies of $\omega^{n-1}$. The relation $\sim$ and the set $P$ are first order definable in $A$ and therefore automatic by Thm. 2.1.

We further define a binary relation $R$ on $\Sigma^{*}$ by

$$
R=\left\{(u, v) \in P \times \Sigma^{*}|| u|=| v \mid \text { and }[u] \cap v \Sigma^{*} \text { has order type } \omega^{n-1}\right\} .
$$

Since any finite partition of a well-ordering of type $\omega^{n-1}$ contains a part of order type $\omega^{n-1}$, for every $u \in P$ there exists a $v \in \Sigma^{*}$ such that $(u, v) \in R$. In addition, $R$ is automatic as it is first order definable in the automatic structure $\left(\Sigma^{*} ; A, \leq_{A}, \sim, P, \equiv, \preceq\right)$, where $\equiv$ and $\preceq$ are the same-length and prefix relations, respectively. Similarly, $[u] \cap v \Sigma^{*}$ is regular for all $(u, v) \in R$.

Since $\otimes R$ is an infinite regular set and due to a pumping argument, there are words $p, q, r, \tilde{p}, \tilde{q}, \tilde{r} \in \Sigma^{*}$ with $|p|=|\tilde{p}|$ and $|q|=|\tilde{q}|>|r|=|\tilde{r}|$ such that $\left(p q^{x} r, \tilde{p} \tilde{q}^{x} \tilde{r}\right) \in R$ for each $x \geq 0$. Let $\eta$ be a morphism into a finite semigroup $S$ which simultaneously recognises $\leq_{A}$ and $\sim$. Pick an idempotency exponent $M \geq 1$ of $S$. We define presentable maps $g: \mathbb{N} \rightarrow P$ and $\tilde{g}: \mathbb{N} \rightarrow \Sigma^{*}$ by

$$
g(x)=p q^{M \cdot(2 x-1)} r \quad \text { and } \quad \tilde{g}(x)=\tilde{p} \tilde{q}^{M \cdot(2 x-1)} \tilde{r} .
$$

Using the idempotency property of $M$, we obtain $\eta(g(x) \otimes g(y))=\eta(g(1) \otimes g(2))$ for all $x, y \in \mathbb{N}$ with $x<y$. This implies that $g$ is an embedding $g: \mathbb{N} \hookrightarrow P$.

For every $x \in \mathbb{N}$ the regular set $B_{x}=[g(x)] \cap \tilde{g}(x) \Sigma^{*} \subseteq A$ has order type $\omega^{n-1}$. We turn the regular set $Z \subseteq \Sigma^{*}$ with $B_{1}=\tilde{g}(1) Z$ into an automatic well-ordering of type $\omega^{n-1}$ by defining $u \leq_{Z} v$ if $\tilde{g}(1) u \leq_{A} \tilde{g}(1) v$. Using the 
idempotency property of $M$ once more yields that for each $x \in \mathbb{N}$ the map $i_{x}: Z \rightarrow B_{x}$ with $i_{x}(u)=\tilde{g}(x) u$ is an isomorphism between well-orderings.

By the induction hypothesis, there is a presentable embedding $h: \mathbb{N}^{n-1} \hookrightarrow Z$. The map $f^{\prime}: \mathbb{N}^{n} \rightarrow \Sigma^{*}$ defined by

$$
f^{\prime}\left(x_{0}, \ldots, x_{n-1}\right)=\tilde{g}\left(x_{0}\right) h\left(x_{1}, \ldots, x_{n-1}\right)
$$

is a presentable embedding $f^{\prime}: \mathbb{N}^{n} \hookrightarrow A$. This completes the induction.

Finally, let $\left(u_{0}, v_{0}, \ldots, u_{n-1}, v_{n-1}, u_{n}\right)$ be a presentation of $f^{\prime}$. Pick a $K \geq 1$ which is divisible by each $\left|v_{i}\right|$, say $K=K_{i} \cdot\left|v_{i}\right|$, and satisfies $K \geq\left|u_{0}\right|+\cdots+\left|u_{n}\right|$. Then the map $f: \mathbb{N}^{n} \rightarrow A$ defined by

$$
f\left(x_{0}, \ldots, x_{n-1}\right)=f^{\prime}\left(K_{0} x_{0}+1, \ldots, K_{n-1} x_{n-1}+1\right)
$$

can be shown to be a $K$-uniformly presentable embedding $f: \mathbb{N}^{n} \hookrightarrow A$.

\subsection{The Automatic Ramsey Degree of $\omega^{n}$}

In this section, we apply Thm. 4.2 to determine the precise value of $d_{R, \mathrm{AUT}}\left(\omega^{n}\right)$ for each $n \geq 0$. In order to expresses these values, we need the following variation of binomial coefficients.

Definition 4.3. For all $n, k \in \mathbb{N}_{0}$ with $k \leq n$ we define $\left\langle\begin{array}{l}n \\ k\end{array}\right\rangle \in \mathbb{N}$ as follows:

(1) $\left\langle\begin{array}{l}n \\ k\end{array}\right\rangle=1$ if $k=0$ or $k=n$,

(2) $\left\langle\begin{array}{l}n \\ k\end{array}\right\rangle=\left\langle\begin{array}{c}n-1 \\ k-1\end{array}\right\rangle+\left\langle\begin{array}{c}n-1 \\ k\end{array}\right\rangle$ if $0<k<n$ and $2 k \neq n$, and

(3) $\left\langle\begin{array}{l}n \\ k\end{array}\right\rangle=\left\langle\begin{array}{c}n-1 \\ k-1\end{array}\right\rangle+\left\langle\begin{array}{c}n-1 \\ k\end{array}\right\rangle+\left\langle\begin{array}{c}n-2 \\ k-1\end{array}\right\rangle$ if $0<k<n$ and $2 k=n$.

Notice that $\left(\begin{array}{l}n \\ k\end{array}\right) \leq\left\langle\begin{array}{l}n \\ k\end{array}\right\rangle$ for all $k \leq n$. This inequality is strict whenever $0<k<n$.

For the rest of this section, we fix some $n \geq 0$ and consider the alphabet $[n]=\{0,1, \ldots, n-1\}$. The lexicographic order on $[n]^{*}$ w.r.t. the reverse order on $[n]$ is denoted by $\leq_{\text {lex }}$. Whenever we use the alphabet $[n]_{\diamond}$, we identify the $\diamond$-symbol with $n$. For $\bar{x} \in \mathbb{N}^{n}$ we define

$$
\langle\bar{x}\rangle=0^{x_{0}} 1^{x_{1}} \cdots(n-1)^{x_{n-1}} \in[n]^{*} .
$$

The set

$$
\left\langle\mathbb{N}^{n}\right\rangle=\left\{\langle\bar{x}\rangle \mid \bar{x} \in \mathbb{N}^{n}\right\}=0^{+} 1^{+} \ldots(n-1)^{+} \subseteq[n]^{*}
$$

ordered by (the restriction of) $\leq_{\operatorname{lex}}$ is an automatic well-ordering of type $\omega^{n}$. The map $\langle\cdot\rangle$ is the unique isomorphism (of well-orderings) between $\mathbb{N}^{n}$ and $\left\langle\mathbb{N}^{n}\right\rangle$.

For all $\bar{x}, \bar{y} \in \mathbb{N}^{n}$ the convolution $\langle\bar{x}\rangle \otimes\langle\bar{y}\rangle$ can be uniquely factorised as $\sigma_{1}^{e_{1}} \cdots \sigma_{k}^{e_{k}}$ with $k \geq 0, \sigma_{1}, \ldots, \sigma_{k} \in[n]_{\diamond}^{2}$ pairwise distinct, and $e_{1}, \ldots, e_{k} \geq 1$. In this situation, the sequence $p(\bar{x}, \bar{y})=\sigma_{1} \ldots \sigma_{k}(n, n)$ is a path through the 2 -dimensional grid from $(0,0)$ to $(n, n)$ using only steps $(0,1),(1,0)$, and $(1,1)$. We call such sequences $n$-paths. We have $\bar{x}<\bar{y}$ if, and only if, $p(\bar{x}, \bar{y})$ contains a step different from $(1,1)$ and the first such is a $(1,0)$-step. We call $n$-paths with this latter property lower $n$-paths. An $n$-path is restricted if the $(1,1)$-step is used only on the main diagonal of the grid. There are precisely $\sum_{i=1}^{n}\left\langle\begin{array}{c}2 i-1 \\ i\end{array}\right\rangle$ restricted lower $n$-paths. 
Theorem 4.4. Let $n \geq 0$. The automatic Ramsey degree $d_{R, \mathrm{AUT}}\left(\omega^{n}\right)$ exists and is given by

$$
d_{R, \mathrm{AUT}}\left(\omega^{n}\right)=\sum_{i=1}^{n}\left\langle\begin{array}{c}
2 i-1 \\
i
\end{array}\right\rangle .
$$

Proof. We prove existence and upper bound separately from the lower bound.

Existence and upper bound. Let $C:[A]^{2} \rightarrow Q$ be an automatic $\omega^{n}$-colouring. By Thm. 4.2, there exists a uniformly presentable embedding $f: \mathbb{N}^{n} \hookrightarrow A$. Consider the $\omega^{n}$-colouring $D:\left[\left\langle\mathbb{N}^{n}\right\rangle\right]^{2} \rightarrow Q$ with $D(\langle\bar{x}\rangle,\langle\bar{y}\rangle)=C(f(\bar{x}), f(\bar{y}))$. Due to the uniform presentability of $f, D$ is automatic as well. Let $\eta$ be a morphism into a finite semigroup $S$ which simultaneously recognises all the $D^{-1}(q)$ and $M \geq 1$ an idempotency exponent of $S$. The set

$$
X_{M}=\left\{\bar{x} \in \mathbb{N}^{n} \mid \forall i \in[0, n-1]: x_{i} \equiv M \quad(\bmod n M)\right\}
$$

has order type $\omega^{n}$ and two useful properties for all $\bar{x}, \bar{y} \in X_{M}$ with $\bar{x}<\bar{y}$ :

(1) The $n$-path $p(\bar{x}, \bar{y})$ is a restricted lower $n$-path.

(2) In the definition of $p(\bar{x}, \bar{y})$ above, each $e_{i}$ is divisible by $M$. Thus, the idempotency properties of $M$ imply $\eta(\langle\bar{x}\rangle \otimes\langle\bar{y}\rangle)=\eta\left(\sigma_{1}^{M} \cdots \sigma_{k}^{M}\right)$, i.e., $p(\bar{x}, \bar{y})$ determines $\eta(\langle\bar{x}\rangle \otimes\langle\bar{y}\rangle)$ and in turn also $C(f(\bar{x}), f(\bar{y}))=D(\langle\bar{x}\rangle,\langle\bar{y}\rangle)$.

Consequently, the regular set $f\left(X_{M}\right) \subseteq A$ is $\sum_{i=1}^{n}\left\langle\begin{array}{c}2 i-1 \\ i\end{array}\right\rangle$-chromatic in $C$.

Lower bound. Let $Q$ be the set of restricted lower $n$-paths. The sets

$$
X_{1}=\left\{\bar{x} \in \mathbb{N}^{n} \mid \forall i \in[0, n-1]: x_{i} \equiv 1 \quad(\bmod n)\right\}
$$

and $\left\langle X_{1}\right\rangle \subseteq\left\langle\mathbb{N}^{n}\right\rangle$ have order type $\omega^{n}$. Like in property (1) of $X_{M}$ above, we have $p(\bar{x}, \bar{y}) \in Q$ for all $\bar{x}, \bar{y} \in X_{1}$ with $\bar{x}<\bar{y}$. The $\omega^{n}$-colouring $C:\left[\left\langle X_{1}\right\rangle\right]^{2} \rightarrow Q$ with $C(\langle\bar{x}\rangle,\langle\bar{y}\rangle)=p(\bar{x}, \bar{y})$ for $\bar{x}<\bar{y}$ is automatic. Since $|Q|=\sum_{i=1}^{n}\left\langle\begin{array}{c}2 i-1 \\ i\end{array}\right\rangle$, it remains to show that for any regular subset $B \subseteq\left\langle X_{1}\right\rangle$ of order type $\omega^{n}$ and all $\pi \in Q$ there are $u, v \in B$ with $u<v$ and $C(u, v)=\pi$.

Therefore, consider such $X$ and $\pi$. By Thm. 4.2, there exists a uniformly presentable embedding $f: \mathbb{N}^{n} \hookrightarrow B$. Moreover, there are $\bar{x}, \bar{y} \in \mathbb{N}^{n}$ with $\bar{x}<\bar{y}$ and $\pi=(\langle\bar{x}\rangle \otimes\langle\bar{y}\rangle)(n, n)$. Finally, one can show that with $\overline{1}=(1, \ldots, 1) \in \mathbb{N}^{n}$ we have $C(f(n \cdot \bar{x}+\overline{1}), f(n \cdot \bar{y}+\overline{1}))=\pi$.

Remark 4.5. The remark after Def. 4.3 implies that $d_{R}\left(\omega^{n}\right)<d_{R, \mathrm{AUT}}\left(\omega^{n}\right)$ for $n \geq 2$. This is caused by the following reasons. In the proof of the lower bound above, you can find a non-regular subset $B \subseteq\left\langle X_{1}\right\rangle$ such that $C(B)$ is the set of restricted lower $n$-paths in which the $(1,1)$-steps form an initial segment. There are precisely $d_{R}\left(\omega^{n}\right)$ such $n$-paths. However, for regular sets $B$ you cannot avoid using $(1,1)$-steps after other steps as they provide more structure.

Using the same techniques, one can show a bipartite analogue of Thm. 4.4.

Theorem 4.6. Let $m, n \geq 0$. The automatic Ramsey degree $d_{R, \mathrm{AUT}}\left(\omega^{m}, \omega^{n}\right)$ exists and is given by

$$
d_{R, \mathrm{AUT}}\left(\omega^{m}, \omega^{n}\right)=\left(\begin{array}{c}
m+n \\
m
\end{array}\right)
$$




\subsection{The Automatic Ramsey Degree of Arbitrary Ordinals $\alpha<\omega^{\omega}$}

Theorem 4.7. Let $\alpha<\omega^{\omega}$ be an ordinal and $\alpha=\omega^{n_{1}}+\cdots+\omega^{n_{r}}$ with $r \geq 0$ and $n_{1} \geq \cdots \geq n_{r} \geq 0$ its Cantor normal form. The automatic Ramsey degree $d_{R, \mathrm{AUT}}(\alpha)$ exists and is given by

$$
d_{R, \mathrm{AUT}}(\alpha)=\sum_{i=1}^{r} d_{R, \mathrm{AUT}}\left(\omega^{n_{i}}\right)+\sum_{i=1}^{r} \sum_{j=i+1}^{r} d_{R, \mathrm{AUT}}\left(\omega^{n_{i}}, \omega^{n_{j}}\right) .
$$

Proof. Let $\mu(\alpha)$ denote the sum on RHS above. Again, we prove existence/upper bound and lower bound separately.

Existence and upper bound. Let $C$ be an automatic $\alpha$-colouring on $A$. There is a unique decomposition $A=A_{1}+\ldots+A_{r}$ such that each $A_{i}$ has order type $\omega^{n_{i}}$. All the $A_{i}$ are regular. We construct regular subsets $B_{i, r} \subseteq \cdots \subseteq B_{i, 1} \subseteq A_{i}$ of order type $\omega^{n_{i}}$ for $i=1, \ldots, r$ in several stages. For $i=1, \ldots, r$ choose $B_{i, 1} \subseteq A_{i}$ by Thm. 4.4 such that $\left|C\left(B_{i, 1}\right)\right| \leq d_{R, \mathrm{AUT}}\left(\omega^{n_{i}}\right)$. For $i=1, \ldots, r$ and $j=i+1, \ldots, r$ choose $B_{i, j} \subseteq B_{i, j-1}$ and $B_{j, i+1} \subseteq B_{j, i}$ by Thm. 4.6 such that $\left|C\left(B_{i, j} \times B_{j, i+1}\right)\right| \leq d_{R, \mathrm{AUT}}\left(\omega^{n_{i}}, \omega^{n_{j}}\right)$. Finally, the set $B=B_{1, r}+\cdots+B_{r, r} \subseteq A$ is regular, has order type $\alpha$, and satisfies

$$
|C(B)| \leq \sum_{i=1}^{r}\left|C\left(B_{i, r}\right)\right|+\sum_{i=1}^{r} \sum_{j=i+1}^{r}\left|C\left(B_{i, r} \times B_{j, r}\right)\right| \leq \mu(\alpha) .
$$

Lower bound. For $1 \leq i \leq r$ let $C_{i}:\left[A_{i}\right]^{2} \rightarrow Q_{i}$ be (a slight modification of) the automatic $\omega^{n_{i}}$-colouring proving the lower bound on $d_{R \text {, AUT }}\left(\omega^{n_{i}}\right)$. Due to (the actual proof of) Thm. 4.4, for $1 \leq i<j \leq r$ there exists an automatic $\left(\omega^{n_{i}}, \omega^{n_{j}}\right)$-colouring $C_{i, j}: A_{i} \times A_{j} \rightarrow Q_{i, j}$ which shows the lower bound on $d_{R, \mathrm{AUT}}\left(\omega^{n_{i}}, \omega^{n_{j}}\right)$. W.l.o.g. all the sets $Q_{i}$ and $Q_{i, j}$ are mutually disjoint. Thus, their union $Q$ has size $\mu(\alpha)$. The well-ordering $A=A_{1}+\ldots+A_{r}$ is automatic and has type $\alpha$. We define an automatic $\alpha$-colouring $C:[A]^{2} \rightarrow Q$ by $C(u, v)=C_{i}(u, v)$ if there is an $i$ such that $u, v \in A_{i}$ and $C(u, v)=C_{i, j}(u, v)$ if there are $i<j$ such that $u \in A_{i}$ and $v \in A_{j}$. For every regular subset $B \subseteq A$ with order type $\alpha$ all the sets $A_{i} \cap B$ are regular and have order type $\omega^{n_{i}}$. Thus, $C(B)=Q$ and hence $|C(B)|=\mu(\alpha)$.

Since all constructions employed throughout this section are effective, we obtain the following result which states that Thm. 4.7 is effective.

Theorem 4.8. Given an automatic presentation of a colouring $C:[A]^{2} \rightarrow Q$, one can compute the following:

(1) $d_{R, \mathrm{AUT}}(\alpha)$, where $\alpha$ is the order type of $A$,

(2) a subset $D \subseteq Q$ of size at most $d_{R, \mathrm{AUT}}(\alpha)$, and

(3) a finite automaton recognising a D-chromatic subset $B \subseteq A$ of order type $\alpha$. 


\section{The Computable Case}

The reader can find the needed notions of computability theory in [16]. We call a colouring $C:[V]^{2} \rightarrow F$ computable if $\left(V ; \leq_{V}\right)$ is a computable ordinal and for each $i \in F$, the preimage $C^{-1}(i)$ is a computable set. Let COMP be the class of computable colourings.

Theorem 5.1 (Specker $[17])$. For any $k \in \mathbb{N},(\omega:$ COMP $) \nrightarrow \rightarrow(\omega: \text { COMP })_{k, 1}$.

It is therefore a natural question whether the polychromatic version of Ramsey's theorem holds in the computable case. We remark in this section that polychromatic Ramsey's theorem also fails for computable colourings. Recall that $\Sigma_{1}$ denotes the class of c.e. sets.

Theorem 5.2. For any $k \in \mathbb{N}$, we have $(\omega: \operatorname{COMP}) \not \rightarrow\left(\omega: \Sigma_{1}\right)_{k+1, k}$.

The proof is conceptually similar to Jockusch's proof of Thm. 5.1 in [8]. For this, one needs the following notion.

Definition 5.3. $A$ set $A \subseteq \mathbb{N}$ is bi-immune if it is infinite and neither $A$ nor $\mathbb{N} \backslash A$ contains an infinite $\Sigma_{1}$ subset. $A k$-immune set partition is a partition $\mathbb{N}=A_{1} \cup \cdots \cup A_{k}$ of the natural numbers such that each $A_{i}$ is bi-immune.

The proof of Prop. 5.4 uses a standard priority argument with finite injury and we include it in Appendix C.

Proposition 5.4. For every $k \in \mathbb{N}$, there exists a $k$-immune set partition $A_{1} \cup \cdots \cup A_{k}$ where each $A_{i}$ is a $\Delta_{2}$ set.

Proof (Proof of Thm. 5.2). Take a $k$-immune set partition $A_{1} \cup \cdots \cup A_{k}$ where each $A_{i}$ is $\Delta_{2}$ as stipulated by Prop. 5.4. By the limit lemma ${ }^{4}$ each set $A_{i}$ is limit computable. In other words there is a computable set $X_{i} \subseteq \mathbb{N}^{2}$ such that $x \in A_{i}$ if and only if $\exists t \forall s \geq t: X_{i}(x, s)$. We define a colouring $C:[\mathbb{N}]^{2} \rightarrow[1, k]$ such that

$$
C(x, s)= \begin{cases}\min \left\{i \mid X_{i}(x, s)\right\} & \text { if } \exists i: X_{i}(x, s) \\ 1 & \text { otherwise. }\end{cases}
$$

Take any $(k-1)$-chromatic infinite set $H \subseteq \mathbb{N}$. Assume there is $x \in H \cap A_{i}$ for $i \in[1, k]$. There is some $t>x$ such that $\forall s \geq t: X_{i}(x, s)$. In particular there is some $y \in H$ such that $y>t$. This means that $C(x, y)=i \in C(H)$. We conclude that for some $i \in[1, k], H \cap A_{i}=\emptyset$. However this means that $H \subseteq \mathbb{N} \backslash A_{i}$ and cannot be a $\Sigma_{1}$ set.

\section{Final Remarks}

This paper presents an explicit expression for computing the Ramsey degree of ordinals $\alpha<\omega^{\omega}$ and establishes the automatic version of this result. Below, we present some questions that came up but remained unanswered.

\footnotetext{
${ }^{4}$ See Appendix $\mathrm{C}$ for the statement of the limit lemma.
} 
(1) Provided that an automatic $\alpha$-colouring admits a regular, $D$-chromatic set of order type $\alpha$, can one compute a finite automaton recognising such a set?

(2) Extend Jockusch's theorems [8, Cor.3.2,Thm.4.2] to ordinals $\alpha<\omega^{\omega}$. In other words, does there exist an $n$ such that $\Pi_{n}$ contains a $d_{R}(\alpha)$-chromatic set of order type $\alpha$ in any computable $\alpha$-colouring?

(3) Does Galvin's result on the rationals mentioned in Section 1 hold in the automatic case?

\section{References}

1. A. Blumensath and E. Grädel. Automatic structures. In LICS 2000, pages 51-62. IEEE Computer Society, 2000.

2. D. Conlon. A new upper bound for the bipartite ramsey problem. Journal of Graph Theory, 58(4):351-356, 2008.

3. C. Delhommé. Automaticité des ordinaux et des graphes homogènes. Comptes Rendus Mathematique, 339(1):5-10, 2004.

4. S. Eilenberg. Automata, Languages, and Machines, volume 58 of Pure and Applied Mathematics. Academic Press, 1974.

5. P. Erdős, A. Hajnal, A. Máté, and R. Rado. Combinatorial set theory: Partition relations for cardinals, volume 106 of Studies in Logic. North-Holland, 1984.

6. P. Erdős and R. Rado. A partition calculus in set theory. Bull. Amer. Math. Soc., 62(5):427-489, 1956.

7. A. Hajnal and P. Komjáth. A strongly non-Ramsey order type. Combinatorica, $17(3): 363-367,1997$.

8. C. G. Jockusch. Ramsey's theorem and recursion theory. Journal of Symbolic Logic, 37(2):268-280, 1972.

9. B. Khoussainov and A. Nerode. Automatic presentations of structures. In $L C C$ 1994, volume 960 of $L N C S$, pages 367-392. Springer, 1994.

10. B. Khoussainov, S. Rubin, and F. Stephan. Automatic linear orders and trees. ACM Trans. Comp. Logic, 6(4):675-700, 2005.

11. D. Kuske. (Un) countable and (non)effective versions of Ramsey's theorem, volume 558 of Contemporary Mathematics, pages 467-487. Am. Math. Soc., 2011.

12. M. Pouzet and N. Sauer. Edge partitions of the rado graph. Combinatorica, 16(4):505 - 520, 1996.

13. F. P. Ramsey. On a problem of formal logic. Proc. London Math. Soc., 30:264-286, 1930.

14. J. G. Rosenstein. Linear Orderings. Academic Press, 1982.

15. S. Rubin. Automata presenting structures: A survey of the finite string case. Bulletin of Symbolic Logic, 14(2):169-209, 2008.

16. R. Soare. Recursively Enumerable Sets and Degrees: A study of computable functions and computably generated sets. Perspectives in Mathematical Logic. Springer, 1987.

17. E. Specker. Ramsey's theorem does not hold in recursive set theory, volume 61 of Studies in Logic and the Foundations of Mathematics, pages 439-442. NorthHolland, 1971.

18. S. Todorcevic. Some partitions of three-dimensional combinatorial cubes. Journal of Combinatorial Theory, Ser. A, 68(2):410-437, 1994.

19. N. Williams. Combinatorial Set Theory. Elsevier, 1977. 


\section{A Missing Proofs in Section 3}

We recall the following terminology which will be used in the proofs below. We view sets also as well-ordered sets, i.e., a set $V$ also denotes a well-ordering $\left(V, \leq_{V}\right)$. By " $V$ has order type $\alpha$ " we mean " $\left(V, \leq_{V}\right)$ has order type $\alpha$ ". By $U+V$ we mean the sum of the well-orderings $\left(U, \leq_{U}\right)+\left(V, \leq_{V}\right)$. If $U \subseteq V$ then we assume the ordering on $U$ is the same as the ordering on $V$ restricted to $U$. For two subsets $U_{1}, U_{2} \subseteq V, U_{1} \ll U_{2}$ denotes the fact that $u_{1}<u_{2}$ for all $u_{1} \in U_{1}$ and $u_{2} \in U_{2}$. We use " $u<v \in V$ " as an abbreviation for " $u, v \in V$ with $x<y$ ". We view $\mathbb{N}^{n}$ where $n \geq 0$ as the canonical representation of $\omega^{n}$.

Lemma A.1. Suppose $A$ has order type $\omega^{n}$ for some $n \in \mathbb{N}_{0}$, and $A=B_{1} \cup$ $\cdots \cup B_{k}$ for some $k \in \mathbb{N}$. There is an $\ell \in[1, k]$ such that $B_{\ell}$ has order type $\omega^{n}$.

Proof. We proceed by induction on $n$. The claim is trivial for $n=0$. Assume $n>0$. Without loss of generality we assume that $A=\mathbb{N}^{n}$. For any $i \in \mathbb{N}$, $\{i\} \times \mathbb{N}^{n-1}$ has order type $\omega^{n-1}$. Furthermore, we have

$$
\{i\} \times \mathbb{N}^{n-1}=\left(\{i\} \times \mathbb{N}^{n-1} \cap B_{1}\right) \cup \cdots \cup\left(\{i\} \times \mathbb{N}^{n-1} \cap B_{k}\right) .
$$

By the inductive hypothesis, for any $i \in \mathbb{N}$ there is some $\ell_{i} \in[1, k]$ such that $\{i\} \times \mathbb{N}^{n-1} \cap B_{\ell_{i}}$ has order type $\omega^{n-1}$.

By the pigeonhole principle, there is $\ell \in[1, k]$ such that the set $I=\{i \in \mathbb{N} \mid$ $\left.\ell_{i}=\ell\right\}$ is infinite. Hence the set $\left(I \times \mathbb{N}^{n-1}\right) \cap B_{\ell}$ has order type $\omega^{n}$. Since

$$
\left(I \times \mathbb{N}^{n-1}\right) \cap B_{\ell} \subseteq B_{\ell} \subseteq \mathbb{N}^{n},
$$

the set $B_{\ell}$ also has order type $\omega^{n}$.

Below we use $\ell(m, n)$, where $m, n \in \mathbb{N}_{0}$, to denote $\left(\begin{array}{c}m+n \\ m\end{array}\right)$.

Lemma A.2. For all $m, n \in \mathbb{N}_{0}$ we have

$$
\forall k \in \mathbb{N}:\left(\omega^{m}, \omega^{n}\right) \rightarrow\left(\omega^{m}, \omega^{n}\right)_{k, \ell(m, n)} .
$$

Proof. It is sufficient to prove the case when $m \leq n$. We first consider the case when $m=0$. Take a $\left(1, \omega^{n}\right)$-colouring $C:\{x\} \times A \rightarrow[1, k]$. Let $B_{i}=\{y \in A \mid$ $C(x, y)=i\}$ where $1 \leq i \leq k$. By Lemma A.1, there is a $d \in[1, k]$ such that $B_{d}$ has order type $\omega^{n}$. Thus $\left(\{x\}, B_{d}\right)$ forms a 1 -chromatic pair of type $\left(1, \omega^{n}\right)$.

The proof proceeds by induction. Take $m \leq n \in \mathbb{N}$ and suppose the lemma holds for all $m^{\prime}<n^{\prime} \in \mathbb{N}_{0}$ where $m^{\prime}+n^{\prime}<m+n$ or $m^{\prime}=0$. We take an $\left(\omega^{m}, \omega^{n}\right)$-colouring $C: N_{1} \times N_{2} \rightarrow[1, k]$. Using a back-and-forth argument, we construct a pair $(X, Y) \subseteq\left(N_{1}, N_{2}\right)$ of type $\left(\omega^{m}, \omega^{n}\right)$. The construction proceeds by stages and defines a sequence of pairs

$$
\left(N_{1}, N_{2}\right)=\left(S_{0}, T_{0}\right) \supseteq\left(S_{1}, T_{1}\right) \supseteq\left(S_{2}, T_{2}\right) \supseteq \cdots
$$

where each $\left(S_{i}, T_{i}\right)$ has order type $\left(\omega^{m}, \omega^{n}\right)$. At every stage $i \geq 1$ we define a pair of sets $\left(X_{i}, Y_{i}\right) \subseteq\left(S_{i-1}, T_{i-1}\right)$ of order type $\left(\omega^{m-1}, \omega^{n-1}\right)$ such that

$$
X_{i-1} \ll X_{i} \ll S_{i} \text { and } Y_{i-1} \ll Y_{i} \ll T_{i} \text {. }
$$


Furthermore, the construction makes the pair $\left(X_{i}, T_{i} \cup Y_{i}\right) \ell(m-1, n)$-chromatic and the pair $\left(S_{i}, Y_{i}\right) \ell(m, n-1)$-chromatic. In other words, there are sets $D_{i}, E_{i} \subseteq$ $[1, k]$, where $\left|D_{i}\right| \leq \ell(m-1, n)$ and $\left|E_{i}\right| \leq \ell(m, n-1)$, such that

$$
C\left(X_{i}, Y_{i} \cup T_{i}\right) \subseteq D_{i} \quad \text { and } \quad C\left(S_{i}, Y_{i}\right) \subseteq E_{i} .
$$

Eventually we let $X=\bigcup_{i \in \mathbb{N}} X_{i}$ and $Y=\bigcup_{i \in \mathbb{N}} Y_{i}$. Note that (1) implies that $(X, Y)$ has order type $\left(\omega^{m}, \omega^{n}\right)$.

We now describe the stage-wise construction. At stage 0 , let $S_{0}=N_{1}$ and $T_{0}=N_{2}$. Let $i>0$ and suppose we have defined $S_{i-1}$ and $T_{i-1}$ as above. Since $S_{i-1}$ has order type $\omega^{m}$, we can write it as a sum of sub-orderings $U_{1}+U_{2}+\cdots$ where each $U_{j}$ has order type $\omega^{m-1}$.

Applying the inductive hypothesis on $\left(U_{1}, T_{i-1}\right)$ we obtain an $\ell(m-1, n)$ chromatic pair $\left(X_{i}, V\right) \subseteq\left(U_{1}, T_{i-1}\right)$ of type $\left(\omega^{m-1}, \omega^{n}\right)$. We let $D_{i}$ be any $\ell(m-$ $1, n)$-element subset of $[1, k]$ that contains every element of $C\left(X_{i}, V\right)$.

Since $V$ has order type $\omega^{n}$, we can write it as a sum $V_{1}+V_{2}+\cdots$ where each $V_{j}$ has order type $\omega^{n-1}$. Let $U=\bigcup_{j>2} U_{j}$. Applying the inductive hypothesis on $\left(U, V_{1}\right)$, we obtain an $\ell(m, n-1)$-chromatic pair $\left(S_{i}, Y_{i}\right) \subseteq\left(U, V_{1}\right)$ of type $\left(\omega^{m}, \omega^{n-1}\right)$. We let $E_{i}$ be any $\ell(m, n-1)$-element subset of $[1, k]$ that contains every element of $C\left(S_{i}, Y_{i}\right)$. Finally we set $T_{i}$ as $\bigcup_{j \geq 2} V_{j}$. This finishes the construction at stage $i$. Note that (1) clearly holds for stage $i$.

By the pigeonhole principle, there are subsets $D, E \subseteq[1, k]$ where $|D|=$ $\ell(m-1, n),|E|=\ell(m, n-1)$ such that the set $I=\left\{i \in \mathbb{N} \mid D_{i}=D\right\}$ and the set $J=\left\{j \in \mathbb{N} \mid E_{j}=E\right\}$ are both infinite. By (2), for any $i \in I$ and $j \in J$, we have

$$
i \leq j \Rightarrow C\left(X_{i}, Y_{j}\right) \subseteq D \text { and } j<i \Rightarrow C\left(X_{i}, Y_{j}\right) \subseteq E .
$$

Therefore the two sets

$$
H_{1}=\bigcup_{i \in I} X_{i} \quad \text { and } \quad H_{2}=\bigcup_{j \in J} Y_{j}
$$

form a $(D \cup E)$-chromatic pair $\left(H_{1}, H_{2}\right) \subseteq\left(N_{1}, N_{2}\right)$ of order type $\left(\omega^{m}, \omega^{n}\right)$, where

$$
|D \cup E| \leq \ell(m-1, n)+\ell(m, n-1)=\ell(m, n) .
$$

Our next goal is to show that for any $n \in \mathbb{N}_{0}$, we can guarantee in any $\omega^{n}$ colouring there exists a $\mu$-chromatic subset of type $\omega^{n}$, where $\mu$ is a fixed number depending only on $n$.

Lemma A.3. Suppose $A$ has order type $\omega^{n+1}$ for some $n \geq 0$ and $A=B_{1}+$ $B_{2}+\ldots$ where each $B_{i}$ has order type $\omega^{n}$. Take $U \subseteq A$ with order type $\omega^{n+1}$. Then there exist infinitely many $i \in \mathbb{N}$ such that $B_{i} \cap U$ has order type $\omega^{n}$.

Proof. Without loss of generality we assume $A=\mathbb{N}^{n+1}$ and each $B_{i}=\{i\} \times \mathbb{N}^{n}$. For $i \in \mathbb{N}$ let $U_{i}=U \cap B_{i}$. For the sake of contradiction we assume that the set $I=\left\{i \mid U_{i}\right.$ has order type $\left.\omega^{n}\right\}$ is finite. For every $j \notin I$ there is some $k \in \mathbb{N}$ 
such that the order type of $U_{i}$ is smaller than $\omega^{n} \cdot k$. Let $k_{j}$ be the least such $k$. Therefore

$$
\begin{aligned}
U=U_{1}+U_{2}+\ldots & <\sum_{i \in I} U_{i}+\sum_{j \notin I} \omega^{n-1} \cdot k_{j} \\
& <\omega^{n} \cdot|I|+\omega^{n-1} \cdot \omega \\
& <\omega^{n} \cdot(|I|+1) .
\end{aligned}
$$

This contradicts the assumption that $U$ has order type $\omega^{n+1}$.

In the following we let $\mu(n)=\sum_{1 \leq i \leq n}\left(\begin{array}{c}2 i-1 \\ i\end{array}\right)$.

Lemma A.4. For all $n \in \mathbb{N}_{0}$, we have

$$
\forall k \in \mathbb{N}: \omega^{n} \rightarrow\left(\omega^{n}\right)_{k, \mu(n)} .
$$

The proof of Lemma A.4 uses the following terminology.

(i) Take $n \geq 0$ and two sets $N_{1}, N_{2}$ of order type $\omega^{n}$. Let $C: N_{1} \times N_{2} \rightarrow[1, k]$ be an $\left(\omega^{n}, \omega^{n}\right)$-colouring. As stipulated by Lemma A.2, we use

$$
\left(A_{1}\left(N_{1}, N_{2}\right), A_{2}\left(N_{1}, N_{2}\right)\right) \subseteq\left(N_{1}, N_{2}\right)
$$

to denote the pair of sets of type $\left(\omega^{n}, \omega^{n}\right)$ that is $\ell(n, n)$-chromatic.

(ii) Take $m, n \in \mathbb{N}_{0}, M$ with order type $\omega^{m}$, and infinitely many sets $N_{1}, N_{2}, \ldots$ each of order type $\omega^{n}$. Let $N=N_{1}+N_{2}+\cdots$ and $C: M \times N \rightarrow[1, k]$ be an $\left(\omega^{m}, \omega^{n+1}\right)$-colouring. By Lemma A.2 there is an $\ell(m, n+1)$-chromatic pair of subsets $(U, V) \subseteq(M, N)$ with order type $\left(\omega^{m}, \omega^{n+1}\right)$. By Lemma A.3 the set $I=\left\{i \mid V \cap N_{i}\right.$ has order type $\left.\omega^{n}\right\}$ is infinite. List all elements of $I$ as $i_{1}<i_{2}<\cdots$. For $j \in \mathbb{N}_{0}$ we define

$$
B_{j}\left(M,\left(N_{i}\right)_{i \in \mathbb{N}}\right)= \begin{cases}U & \text { if } j=0 ; \\ V \cap N_{i_{j}} & \text { otherwise. }\end{cases}
$$

The following holds by the above definition:

$$
\forall j \in \mathbb{N} \exists \ell \geq j: B_{j}\left(M,\left(N_{i}\right)_{i \in \mathbb{N}}\right) \subseteq N_{\ell} .
$$

(iii) We fix the bijection $f: \mathbb{N}_{<}^{2} \cup\{(0,1)\} \rightarrow \mathbb{N}_{0}$ such that

$$
f(i, j)=\frac{2 i+j^{2}-3 j+2}{2} .
$$

We may view $f$ as an enumeration of all elements in $\mathbb{N}_{<}^{2} \cup\{(0,1)\}$ where the $i^{\text {th }}$ enumerated pair is $f^{-1}(i)$. The first few pairs enumerated in this way are:

$$
(0,1),(1,2),(1,3),(2,3),(1,4),(2,4),(3,4), \ldots
$$

We single out the following property of the function $f$ : For $\left(i_{1}, j_{1}\right),\left(i_{2}, j_{2}\right) \in$ $\mathbb{N}_{<}^{2} \cup\{(0,1)\}$ and $j \in \mathbb{N}$ :

$$
f\left(i_{1}, j_{1}\right)<f(1, j)<f\left(i_{2}, j_{2}\right) \Rightarrow j_{1}<j \leq j_{2} .
$$


Proof (Proof of Lemma A.4). For $n=0$ the statement is trivial; for $n=1$ the statement is simply Ramsey's theorem. The proof proceeds by induction on $n$. Take $n>1$ and an $\omega^{n}$-colouring $C: V \rightarrow[1, k]$. W.l.o.g., we assume $V=\mathbb{N}^{n}$. We construct a $\mu(n)$-chromatic set $H \subseteq V$ of order type $\omega^{n}$. The construction contains two parts. In the first part, we construct infinite sets $K_{1}, K_{2}, \ldots$ by defining sequence of sets $\left(K_{i, j}\right)_{i<j \in \mathbb{N}}$ and making $K_{i}=\bigcup_{j>i} K_{i, j}$. In the second part, we use the results of the first part to construct the desired set $H$. Below we present the main part of the construction.

We start with the first part of the construction. We will make sure that $K_{i, j} \ll K_{i, j^{\prime}}$ whenever $j<j^{\prime}$ and each $K_{i, j}$ has order type $\omega^{n-2}$. Thus $K_{i}$ has order type $\omega^{n-1}$.

Stage 0 . By the inductive hypothesis for every $i \in \mathbb{N}$ there is a $\mu(n-1)$-chromatic subset $N_{i} \subseteq\{i\} \times \mathbb{N}^{n-1}$ of order type $\omega^{n-1}$. Let $\Upsilon_{i} \subseteq[1, k]$ be a $\mu(n-1)$-element set containing all colours in $C\left(N_{i}\right)$. By the pigeonhole principle there is a set $\Upsilon \subseteq[1, k]$ such that the set $I=\left\{i \mid \Upsilon_{i}=\Upsilon\right\}$ is infinite. List $I$ as $i_{1}<i_{2}<\cdots$. For $j \in \mathbb{N}$, let $X_{j, 0}=N_{i_{j}}$. This ensures

$$
\forall i \in \mathbb{N}: C\left(X_{i, 0}\right) \subseteq \Upsilon
$$

Stage $s=f(i, j)>0$. Suppose we have defined infinite sets $\left(X_{m, s-1}\right)_{m \in \mathbb{N}}$ and $\left(K_{i^{\prime}, j^{\prime}}\right)_{0<f\left(i^{\prime}, j^{\prime}\right)<s}$. We assume the following inductive hypothesis:

(I1) $X_{m, s-1}$ has order type $\omega^{n-1}$ where $m \in \mathbb{N}$;

(I2) $X_{m, s-1} \ll X_{m^{\prime}, s-1}$ whenever $m<m^{\prime}$;

(I3) $K_{i^{\prime}, j^{\prime}}$ has order type $\omega^{n-2}$ where $0<f\left(i^{\prime}, j^{\prime}\right)<s$;

(I4) $\exists x \in X_{i^{\prime}, s-1} \forall y \in K_{i^{\prime}, j^{\prime}}: y<_{n} x$ for $0<f\left(i^{\prime}, j^{\prime}\right)<s$.

We now define infinite sets $\left(X_{m, s}\right)_{m \in \mathbb{N}}$ of order type $\omega^{n-1}$ and the set $K_{i, j} \subseteq X_{i, s}$.

Let $X_{i, s}=A_{1}\left(X_{i, s-1}, X_{j, s-1}\right)$. By (I1) $X_{i, s}$ has order type $\omega^{n-1}$. By (I3) all $K_{i, j^{\prime}}$ where $j^{\prime}<j$ have been defined and have order type $\omega^{n-2}$. By (I4), the set

$$
\widehat{X}=X_{i, s} \backslash \bigcup_{j^{\prime} \in[1, j-i 1]} K_{i, j^{\prime}}
$$

has order type $\omega^{n-1}$. Thus we may write $\widehat{X}$ as $\widehat{X}_{1}+\widehat{X}_{2}+\ldots$ where each $\widehat{X}_{m}$ has order type $\omega^{n-2}$. The construction then performs the following:

1. Let $X_{j, s}=A_{2}\left(X_{i, s-1} \backslash K_{i, j-1}, X_{j, s-1}\right)$.

2. Let $K_{i, j}=B_{0}\left(\widehat{X}_{1},\left(X_{j^{\prime}, s-1}\right)_{j^{\prime}>j}\right)$.

3. For any $\ell>j$, let $X_{\ell, s}=B_{\ell-j}\left(\widehat{X}_{1},\left(X_{j^{\prime}, s-1}\right)_{j^{\prime}>j}\right)$.

4. For any $\ell<j$ and $\ell \neq i$, let $X_{\ell, s}=X_{\ell, s-1}$.

This finishes the construction at stage $s$. Note that the inductive hypotheses (I1)-(I4) all hold for $s$. Furthermore, we have:

$$
\begin{aligned}
& \left|C\left(X_{i, s}, X_{j, s}\right)\right| \leq \ell(n-1, n-1), \quad \text { and } \\
& \left|C\left(K_{i, j}, \bigcup_{j^{\prime}>j} X_{j^{\prime}, s}\right)\right| \leq \ell(n-2, n) .
\end{aligned}
$$


For $i \in \mathbb{N}$ let $K_{i}=\bigcup_{j>i} K_{i, j}$. By (I3), it is clear that $K_{i}$ has order type $\omega^{n-1}$. We make the following claims:

Claim. For any $i \in \mathbb{N}$ we have

(a) for $0 \leq s<f(1, i), X_{i, s} \subseteq X_{j, s-1}$ for some $j \geq i$;

(b) for $s \geq f(1, i), X_{i, s} \subseteq X_{i, s-1}$.

Proof. Take $i \in \mathbb{N}, s \geq 0$. Suppose $f^{-1}(s)=\left(i^{\prime}, j^{\prime}\right) \in \mathbb{N}_{<}^{2} \cup\{(0,1)\}$. For (a), assume $s<f(1, i)$. By $(4), j^{\prime}<i$. This means that at stage $s$, the set $X_{i, s}$ is set to $B_{i-j^{\prime}}\left(\widehat{X}_{1},\left(X_{m, s-1}\right)_{m>j}\right)$ for some $\widehat{X}_{0}$. By (3) there is some $\ell \geq i$ such that $X_{i, s} \subseteq X_{\ell, s-1}$ and (a) is proved. For (b), assume $f(1, i) \leq s$. By (4), $j^{\prime} \geq i$. This means that at stage $s, X_{i, s}$ is either set to a subset of $A_{1}\left(X_{i, s-1}, X_{j^{\prime}, s-1}\right)$, or set to $A_{2}\left(X_{i^{\prime}, s-1}, X_{i, s-1}\right)$, or $X_{i, s-1}$. In any case we have $X_{i, s} \subseteq X_{i, s-1}$. Thus (b) is proved.

Claim. We have $K_{i} \subseteq X_{i, f(1, i)}$ for all $i>1$.

Proof. Take $(i, j) \in \mathbb{N}_{<}^{2}$. The construction ensures that $K_{i, j} \subseteq X_{i, f(i, j)}$. By Claim 1 (b), $X_{i, f(i, j)} \subseteq X_{i, f(1, i)}$. Therefore $K_{i} \subseteq X_{i, f(1, i)}$. The claim is proved.

Claim. For any $(i, j) \in \mathbb{N}_{<}^{2}, K_{i} \ll K_{j}$.

Proof. Take $x \in K_{i}$ and $y \in K_{j}$ for some $i<j$. By Claim $2 x \in X_{i, f(1, i)}$ and $y \in X_{j, f(1, j)}$. Take the $\leq_{n}$-least element $z$ in $X_{j, f(1, i)}$. By (I2) $x<_{n} z$. By Claim $1\left(\right.$ a) $X_{j, f(1, j)} \subseteq X_{j^{\prime}, f(1, i)}$ for some $j^{\prime} \geq j$. Therefore by (I2) $z \leq_{n} y$. Hence $x<_{n} y$.

Claim. For every $(i, j) \in \mathbb{N}_{<}^{2}$ there exist sets of colours $U(i, j)$ and $V(i, j)$ such that for any $\left(i_{1}, j_{1}\right),\left(i_{2}, j_{2}\right) \in \mathbb{N}_{<}^{2}$ where $i_{1}<i_{2}$ we have

(a) If $i_{2} \leq j_{1}$ then $C\left(K_{i_{1}, j_{1}}, K_{i_{2}, j_{2}}\right) \in U\left(i_{1}, i_{2}\right)$;

(b) If $j_{1}<i_{2}$ then $C\left(K_{i_{1}, j_{1}}, K_{i_{2}, j_{2}}\right) \in V\left(i_{1}, j_{1}\right)$.

Proof. For (a), take $j_{1} \geq i_{2}$ and let

$$
U\left(i_{1}, i_{2}\right)=C\left(X_{i_{1}, f\left(i_{1}, i_{2}\right)}, X_{i_{2}, f\left(i_{1}, i_{2}\right)}\right) .
$$

By (6), $\left|U\left(i_{1}, i_{2}\right)\right| \leq \ell(n-1, n-1)$. We have

$$
\begin{aligned}
& f\left(i_{1}, j_{1}\right) \geq f\left(i_{1}, i_{2}\right) \geq f\left(1, i_{2}\right), \quad \text { and } \\
& f\left(i_{2}, j_{2}\right) \geq f\left(i_{2}, i_{2}+1\right)>f\left(i_{1}, i_{2}\right) .
\end{aligned}
$$

By Claim 1(b), we get

$$
\begin{aligned}
& K_{i_{1}, j_{1}} \subseteq X_{i, f\left(i_{1}, j_{1}\right)} \subseteq X_{i, f\left(i_{1}, i_{2}\right)}, \quad \text { and } \\
& K_{i_{2}, j_{2}} \subseteq X_{i_{2}, f\left(i_{2}, j_{2}\right)} \subseteq X_{i_{2}, f\left(i_{1}, i_{2}\right)} .
\end{aligned}
$$

Therefore $C\left(K_{i_{1}, j_{1}}, K_{i_{2}, j_{2}}\right) \subseteq U\left(i_{1}, i_{2}\right)$. 
For (b), take $j_{1}<i_{2}$ and let

$$
V\left(i_{1}, j_{1}\right)=C\left(K_{i_{1}, j_{1}}, \bigcup_{m>j_{1}} X_{m, f\left(i_{1}, j_{1}\right)}\right) .
$$

By (7), $\left|V\left(i_{1}, j_{1}\right)\right| \leq \ell(n-2, n)$. We have

$$
\begin{array}{rlr}
K_{i_{2}, j_{2}} & \subseteq X_{i_{2}, f\left(1, i_{2}\right)} & \\
& \subseteq X_{m, f\left(i_{1}, j_{1}\right)} \text { for some } m>i_{2} . & \text { (by Claim 2) }
\end{array}
$$

Therefore $C\left(K_{i_{1}, j_{1}}, K_{i_{2}, j_{2}}\right) \subseteq V\left(i_{1}, j_{1}\right)$.

Note that we can view $U$ as a colouring that maps $[\mathbb{N}]^{2}$ to the (finite) class of all $\ell(n-1, n-1)$-element subsets of $[1, k]$. By Ramsey's theorem, there is an $\ell(n-1, n-1)$-element set $\Gamma \subseteq[1, k]$ such that there is a $\Gamma$-chromatic infinite subset $J \subseteq \mathbb{N}$ (with respect to $U$ ). By the pigeonhole principle, there is an $\ell(n-2, n)$-element set $\Lambda \subseteq[1, k]$ such that for infinitely many $i \in J$, the set $H_{i}=\{j \mid V(i, j) \subseteq E\}$ is infinite. We define

$$
H=\bigcup_{i \in J, j \in H_{i}} K_{i, j}
$$

By Claim A we get

$$
\left(H, \leq_{n}\right)=\sum_{i \in J} \sum_{j \in H_{i}} K_{i, j} \cong \omega^{n-1} \cdot \omega=\omega^{n} .
$$

Take an edge $\{x, y\} \in[H]^{2}$ where $x<_{n} y$ :

- If there is some $i \in \mathbb{N}^{2}$ with $x, y \in K_{i}$, then by (5) $C(\{x, y\}) \in \Upsilon$.

- If $x \in K_{i_{1}, j_{1}}, y \in K_{i_{2}, j_{2}}$ where $i_{1}<i_{2}$ and $i_{2} \geq j_{1}$, then by Claim 4(a),

$$
C(\{x, y\}) \in U\left(i_{1}, i_{2}\right)=\Gamma .
$$

- If $x \in K_{i_{1}, j_{1}}, y \in K_{i_{2}, j_{2}}$ where $i_{1}<i_{2}$ and $j_{1}<i_{2}$, then by Claim 4(b),

$$
C(\{x, y\}) \in V\left(i_{1}, j_{1}\right)=\Lambda \text {. }
$$

Therefore, $H$ is $(\Upsilon \cup \Gamma \cup \Lambda)$-chromatic, where

$$
|\Upsilon \cup \Gamma \cup \Lambda| \leq \mu(n-1)+\ell(n-1, n-1)+\ell(n-2, n) .
$$

The RHS evaluates to $\mu(n)$ and thus $H \subseteq \mathbb{N}^{n}$ is a $\mu(n)$-chromatic subset of order type $\omega^{n}$.

For $\alpha<\omega^{\omega}$, let

$$
m(\alpha)=\sum_{1 \leq i \leq r} \sum_{1 \leq j \leq n_{i}}\left(\begin{array}{c}
2 j-1 \\
j
\end{array}\right)+\sum_{1 \leq i<j \leq r}\left(\begin{array}{c}
n_{i}+n_{j} \\
n_{i}
\end{array}\right)
$$


Lemma A.5. For any ordinal $\alpha<\omega^{\omega}$

$$
\forall k \in \mathbb{N}: \alpha \rightarrow(\alpha)_{k, m(\alpha)} .
$$

Proof. Take an ordinal $\alpha<\omega^{\omega}$. Write $\alpha$ in its Cantor normal form

$$
\alpha=\omega^{n_{1}}+\cdots+\omega^{n_{r}}
$$

where $r \geq 0$ and $n_{1} \geq \cdots \geq n_{r} \geq 0$. Note that

$$
m(\alpha)=\sum_{1 \leq i \leq r} \mu\left(n_{i}\right)+\sum_{1 \leq i<j \leq r} \ell\left(n_{i}, n_{j}\right) .
$$

We prove by induction on $r$ that $\alpha \rightarrow(\alpha)_{k, m(\alpha)}$ for any $k \in \mathbb{N}$. The claim for $r=0$ is trivial and for $r=1$ it is proved by Lemma A.4.

Suppose $r>1$. Let $C:[V]^{2} \rightarrow[1, k]$ be an $\alpha$-colouring on a set $V$. We write $V$ as $V_{1}+V_{2}$ where $V_{1}$ has order type $\omega^{n_{1}}$ and $V_{2}$ has order type $\beta=\omega^{n_{2}}+\cdots+\omega^{n_{r}}$. By Lemma A.4 there is a $\mu\left(n_{1}\right)$-chromatic subset $E_{1} \subseteq V_{1}$ whose order type is $\omega^{n_{1}}$. By the inductive hypothesis, there is an $m(\beta)$-chromatic subset $E \subseteq V_{2}$ of order type $\beta$. We write $E$ as $E_{2}+\cdots+E_{r}$ where $E_{i}$ has order type $\omega^{\overline{n_{i}}}$ for $i \in[2, n]$.

We inductively define below two sequences of sets:

$$
\begin{aligned}
& E_{1}=F_{1} \supseteq F_{2} \supseteq \ldots \supseteq F_{r} \text { and } \\
& H_{2} \subseteq E_{2}, H_{3} \subseteq E_{3}, \ldots, H_{r} \subseteq E_{r} .
\end{aligned}
$$

Take $i \in[2, n]$. Assume $F_{i-1}$ is defined and has order type $\omega^{n_{1}}$. By Lemma A.2 there is an $\ell\left(n_{1}, n_{i}\right)$-chromatic pair $(X, Y) \subseteq\left(F_{i-1}, E_{i}\right)$ of type $\left(\omega^{n_{1}}, \omega^{n_{i}}\right)$. Set $F_{i}=X$ and $H_{i}=Y$.

Let $H=F_{r} \cup H_{2} \cup \ldots \cup H_{r} \subseteq F_{r} \cup E$. It is clear that

$$
\begin{aligned}
|C(H)| & \leq\left|C\left(F_{r}\right)\right|+|C(E)|+\sum_{2 \leq i \leq r}\left|C\left(F_{r}, H_{i}\right)\right| \\
& \leq m(\beta)+\mu\left(n_{1}\right)+\sum_{2 \leq i \leq r} \ell\left(n_{1}, n_{i}\right) .
\end{aligned}
$$

The RHS evaluates to $m(\alpha)$ and therefore $H \subseteq V$ is an $m(\alpha)$-chromatic subset of order type $\alpha$.

Lemma A.5 shows that $d_{R}(\alpha) \leq m(\alpha)$ for any $\alpha<\omega^{\omega}$. To show that $d_{R}(\alpha)=$ $m(\alpha)$ we need the following definition.

Definition A.6. Let $n \geq 0$. An n-path is a word

$$
\pi=\left(a_{1}, b_{1}\right) \ldots\left(a_{s}, b_{s}\right) \in\{(0,1),(1,0),(1,1)\}^{*}
$$

satisfying $a_{1}+\cdots+a_{s}=b_{1}+\cdots+b_{s}=n$. The set of all $n$-paths resp. those not containing $(1,1)$ is denoted by $P_{n}$ resp. $P_{n}^{N}$. From any path $\pi \in P_{n}$ one obtains a path $\pi^{N} \in P_{n}^{N}$ by replacing each occurrence of $(1,1)$ with $(1,0)(0,1)$. 
Definition A.7. Let $n \geq 0$. For $\bar{x}, \bar{y} \in \mathbb{N}_{<}^{n}$ we define an n-path $p(\bar{x}, \bar{y}) \in P_{n}$ as follows: Let $Z(\bar{x})=\left\{x_{1}, \ldots, x_{n}\right\}, Z(\bar{y})=\left\{y_{1}, \ldots, y_{n}\right\}$, and enumerate the elements of $Z(\bar{x}) \cup Z(\bar{y})$ as $z_{1}<\cdots<z_{s}$. Then

$$
p(\bar{x}, \bar{y})=\left(a_{1}, b_{1}\right) \ldots\left(a_{s}, b_{s}\right),
$$

where $a_{i}, b_{i} \in\{0,1\}$ are defined by $a_{i}=1$ iff $z_{i} \in Z(\bar{x})$ and $b_{i}=1$ iff $z_{i} \in Z(\bar{y})$.

Let $L_{n}$ be the set of all $n$-paths containing a symbol different from $(1,1)$ and such that the first of those symbols is $(1,0)$. For all $\bar{x}, \bar{y} \in \mathbb{N}_{<}^{n}$ we have $\bar{x}<\bar{y}$ if, and only if, $p(\bar{x}, \bar{y}) \in L_{n}$.

Lemma A.8. For all $n \in \mathbb{N}_{0}$, we have

$$
d_{R}\left(\omega^{n}\right)=\sum_{1 \leq i \leq n}\left(\begin{array}{c}
2 i-1 \\
i
\end{array}\right)
$$

Proof. For all $\bar{x}, \bar{y} \in \mathbb{N}_{<}^{n}$ with $\bar{x}<\bar{y}$ the path $p(\bar{x}, \bar{y}) \in P_{n}$ can be uniquely written as $p(\bar{x}, \bar{y})=(1,1)^{n-j} \rho$ with $j \in[1, n]$ and $\rho \in P_{j}$ starting with $(1,0)$. Defining $C(\bar{x}, \bar{y})=(1,1)^{n-j} \rho^{N}$ yields an $\omega^{n}$-colouring $C$ on $\mathbb{N}_{<}^{n}$ with

$$
\operatorname{im}(C)=\bigcup_{1 \leq i \leq n}(1,1)^{n-i}\left((1,0) \Gamma^{*} \cap P_{i}^{N}\right) \quad \text { and } \quad|\operatorname{im}(C)|=\sum_{1 \leq i \leq n}\left(\begin{array}{c}
2 i-1 \\
i
\end{array}\right) .
$$

One can show that each subset $X \subseteq \mathbb{N}_{<}^{n}$ satisfies $C(X)=\operatorname{im}(C)$. This lower bound on $d_{R}\left(\omega^{n}\right)$ matches the upper bound from Thm. A.4.

The bipartite Ramsey degree can be treated using similar ideas.

Theorem 3.6. For all $m, n \in \mathbb{N}_{0}$, we have

$$
d_{R}\left(\omega^{m}, \omega^{n}\right)=\left(\begin{array}{c}
m+n \\
m
\end{array}\right) .
$$

Decomposing ordinals $\alpha<\omega^{\omega}$ into Cantor normal form and combining the colourings from the proofs of Lemma A.8 and Lemma 3.6 in a suitable way yields a lower bound on $d_{R}(\alpha)$ matching the upper bound in Eq. (8) below Lemma A.5.

Theorem 3.3. For all ordinals $\alpha<\omega^{\omega}$, we have

$$
d_{R}(\alpha)=\sum_{1 \leq i \leq r} \sum_{1 \leq j \leq n_{i}}\left(\begin{array}{c}
2 j-1 \\
j
\end{array}\right)+\sum_{1 \leq i<j \leq r}\left(\begin{array}{c}
n_{i}+n_{j} \\
n_{i}
\end{array}\right)
$$

where $\alpha=\omega^{n_{1}}+\cdots+\omega^{n_{r}}$ with $r \geq 0$ and $n_{1} \geq \cdots \geq n_{r} \geq 0$ is the Cantor normal form of $\alpha$. 


\section{B Missing Details of Proofs in Section 4}

\section{B.1 Missing Details for Theorem 4.2}

Theorem 4.2. Let $n \geq 0$. For every automatic well-ordering $A$ of type $\omega^{n}$ there exists a uniformly presentable embedding $f: \mathbb{N}^{n} \hookrightarrow A$.

Within the proof, we need the following.

Lemma B.1. Let $n \geq 0$. There are first-order sentences $\Phi_{\omega^{n}}$ and $\Phi_{<\omega^{n}}$ such that for any well-ordering $A$ we have:

(1) A satisfies $\Phi_{\omega^{n}}$ if, and only if, $A$ has order type $\omega^{n}$.

(2) A satisfies $\Phi_{<\omega^{n}}$ if, and only if, the order type of $A$ is below $\omega^{n}$.

Proof. We first show (1), then (2).

To (1). We proceed by induction on $n$. Clearly, $\omega^{0}$ is characterised by

$$
\Phi_{\omega^{0}}=\exists ! x(x=x) .
$$

In any well-ordering, the formula

$$
\lambda(x)=\forall y(y<x \rightarrow \exists z(y<z<x))
$$

is satisfied by all points without a direct predecessor, i.e., by the limit points and the least element. Among all well-orderings, those of type $\omega$ are characterised by

$$
\Phi_{\omega^{1}}=\exists ! x \lambda(x) \wedge \forall y \exists z(y<z) .
$$

Finally, a well-ordering has order type $\omega^{n}$ for $n \geq 2$ precisely if the set of its limit points (together with the least element) has order type $\omega^{n-1}$. Consequently, we choose

$$
\Phi_{\omega^{n}}=\Phi_{\omega^{n-1} \uparrow_{\lambda}},
$$

i.e., the relativisation of $\Phi_{\omega^{n-1}}$ to $\lambda$.

To (2). The order type of a well-ordering is below $\omega^{n}$ precisely if neither it nor any of its proper initial segments have order type $\omega^{n}$, i.e.,

$$
\Phi_{<\omega^{n}}=\neg \Phi_{\omega^{n}} \wedge \neg \exists x\left(\Phi_{\omega^{n} \Upsilon_{<x}}\right),
$$

where $\Phi_{\omega^{n}} \uparrow_{<x}$ is the relativisation of $\Phi_{\omega^{n}}$ to all $y$ satisfying $y<x$.

The following non-trivial claims remained unproven in the proof of Thm. 4.2.

Claim B.1. The relation $\sim$ and the set $P$ are first order definable in $A$. 
Proof. The relation $\sim$ is defined by the formula

$$
\varphi_{\sim}(x, y)=\Phi_{<\omega^{n-1}} \uparrow_{[x, y)},
$$

i.e., the relativisation of $\Phi_{<\omega^{n-1}}$ to all $z$ satisfying $x \leq z<y$. The set $P$ is defined by

$$
\varphi_{P}(x)=\forall y\left(\varphi_{\sim}(x, y) \rightarrow x \leq y\right)
$$

Claim B.2. Any finite partition of a well-ordering of type $\omega^{n-1}$ contains a part of type $\omega^{n-1}$.

Proof. This follows immediately from [10, Proposition 4.4].

Claim B.3. The relation

$$
R=\left\{(u, v) \in P \times \Sigma^{*}|| u|=| v \mid \text { and }[u] \cap v \Sigma^{*} \text { has order type } \omega^{n-1}\right\} .
$$

is first order definable in $\mathcal{S}=\left(\Sigma^{*} ; A, \leq_{A}, \sim, P, \equiv, \preceq\right)$ and $[u] \cap v \Sigma^{*}$ is regular for all $(u, v) \in R$.

Proof. The relation $R$ is defined by the formula

$$
\varphi_{R}(x, y)=P(x) \wedge x \equiv y \wedge \Omega_{n-1} \uparrow_{\psi(x, y, \cdot)},
$$

where $\Omega_{n-1} \uparrow_{\psi(x, y, \cdot)}$ is the relativisation of $\Omega_{n-1}$ to all $z$ satisfying the formula

$$
\psi(x, y, z)=x \sim z \wedge y \preceq z .
$$

The set $[u] \cap v \Sigma^{*}$ is defined in $\mathcal{S}$ by the same formula $\psi(x, y, z)$ with parameters $x$ any $y$ bound to $u$ and $v$, respectively. Thus, this set is regular by Thm. 2.1.

Claim B.4. For all $x, y \in \mathbb{N}$ with $x<y$ we have

$$
\eta(g(x) \otimes g(y))=\eta(g(1) \otimes g(2))
$$

and $g$ is an embedding $g: \mathbb{N} \hookrightarrow P$.

Proof. For $u, v \in \Sigma^{*}$ we use

$$
\eta\left[\begin{array}{l}
u \\
v
\end{array}\right]
$$

as another way to write $\eta(u \otimes v)$. Let $x, y \in \mathbb{N}$ with $x<y$. We have

$$
\begin{aligned}
& \eta\left[\begin{array}{l}
g(x) \\
g(y)
\end{array}\right]=\eta\left[\begin{array}{ccccc}
p & q^{M \cdot(2 x-1)} & r & \varepsilon & \varepsilon \\
p q^{M \cdot(2 x-1)} & q^{M} & q^{M \cdot(2(y-x)-1)} & r
\end{array}\right] \\
& =\eta\left[\begin{array}{l}
p \\
p
\end{array}\right] \cdot \eta\left[\begin{array}{l}
q \\
q
\end{array}\right]^{M \cdot(2 x-1)} \cdot \eta\left[\begin{array}{c}
r \\
q^{M}
\end{array}\right] \cdot \eta\left[\begin{array}{l}
\varepsilon \\
q
\end{array}\right]^{M \cdot(2(y-x)-1)} \cdot \eta\left[\begin{array}{l}
\varepsilon \\
r
\end{array}\right] \\
& =\eta\left[\begin{array}{l}
p \\
p
\end{array}\right] \cdot \eta\left[\begin{array}{l}
q \\
q
\end{array}\right]^{M} \cdot \eta\left[\begin{array}{c}
r \\
q^{M}
\end{array}\right] \cdot \eta\left[\begin{array}{l}
\varepsilon \\
q
\end{array}\right]^{M} \cdot \eta\left[\begin{array}{l}
\varepsilon \\
r
\end{array}\right] \\
& =\eta\left[\begin{array}{ccccc}
p & q^{M} & r & \varepsilon & \varepsilon \\
p & q^{M} & q^{M} & q^{M} & r
\end{array}\right] \\
& =\eta\left[\begin{array}{l}
g(1) \\
g(2)
\end{array}\right]
\end{aligned}
$$


where the second and fourth equality use that $\left|q^{M}\right| \geq|r|$ and the third equality uses idempotency. Since $\eta$ recognises $\leq_{A}$ this means that $g$ is either strictly increasing or strictly decreasing, depending on which one of $g(1)<g(2)$ or $g(1)>g(2)$ holds true. However, since $P$ has order type $\omega, g$ cannot be strictly decreasing and is hence strictly increasing, i.e., an embedding $g: \mathbb{N} \hookrightarrow P$.

Claim B.5. For each $x \in \mathbb{N}$ the map $i_{x}: Z \rightarrow B_{x}$ with $i_{x}(u)=\tilde{g}(x) u$ is an isomorphism between well-orderings.

Proof. The well-ordering $Z$ was precisely constructed such that $i_{1}$ is an isomorphism. Now, fix an arbitrary $x \in \mathbb{N}$. For all $u \in \Sigma^{*}$ we have

$$
\begin{aligned}
\eta\left[\begin{array}{c}
g(x) \\
\tilde{g}(x) u
\end{array}\right] & =\eta\left[\begin{array}{ccc}
p & q^{M \cdot(2 x-1)} & r \\
\tilde{p} & \tilde{q}^{M \cdot(2 x-1)} & \tilde{r} u
\end{array}\right]=\eta\left[\begin{array}{c}
p \\
\tilde{p}
\end{array}\right] \cdot \eta\left[\begin{array}{c}
q \\
\tilde{q}
\end{array}\right]^{M \cdot\left(2 x_{n}-1\right)} \cdot \eta\left[\begin{array}{c}
r \\
\tilde{r} u
\end{array}\right] \\
& =\eta\left[\begin{array}{c}
p \\
\tilde{p}
\end{array}\right] \cdot \eta\left[\begin{array}{c}
q \\
\tilde{q}
\end{array}\right]^{M} \cdot \eta\left[\begin{array}{c}
r \\
\tilde{r} u
\end{array}\right]=\eta\left[\begin{array}{ccc}
p & q^{M} & r \\
\tilde{p} & \tilde{q}^{M} & \tilde{r} u
\end{array}\right]=\eta\left[\begin{array}{c}
g(1) \\
\tilde{g}(1) u
\end{array}\right],
\end{aligned}
$$

where the second and fourth equality use that $|p|=|\tilde{p}|$ and $|q|=|\tilde{q}|$, and the third equality uses idempotency. For $u \in Z$ we have $g(1) \sim \tilde{g}(1) u$ and since $\eta$ recognises $\sim$, this implies $g(x) \sim \tilde{g}(x) u$ and hence $i_{x}(u)=\tilde{g}(x) u \in B_{x}$, i.e., $i_{x}$ is well-defined. Conversely, for every $w \in B_{x}$ there exists a $u \in \Sigma^{*}$ such that $w=\tilde{g}(x) u$. Since $w \in B_{x}$ we have $g(x) \sim \tilde{g}(x) u$ and hence $g(1) \sim \tilde{g}(1) u$. Thus, $u \in Z$ and $w=i_{x}(u)$, i.e., $i_{x}$ maps onto $B_{x}$. It remains to show that $i_{x}$ is an embedding $i_{x}: Z \hookrightarrow B_{x}$.

For all $u, v \in Z$ we obtain

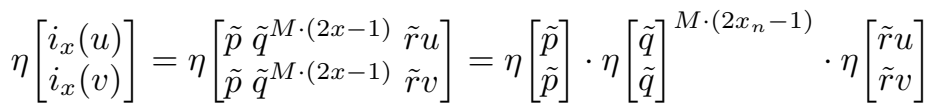

$$
\begin{aligned}
& =\eta\left[\begin{array}{l}
\tilde{p} \\
\tilde{p}
\end{array}\right] \cdot \eta\left[\begin{array}{c}
\tilde{q} \\
\tilde{q}
\end{array}\right]^{M} \cdot \eta\left[\begin{array}{l}
\tilde{r} u \\
\tilde{r} v
\end{array}\right]=\eta\left[\begin{array}{ccc}
\tilde{p} & \tilde{q}^{M} & \tilde{r} u \\
\tilde{p} & \tilde{q}^{M} & \tilde{r} v
\end{array}\right] \eta\left[\begin{array}{l}
i_{1}(u) \\
i_{1}(v)
\end{array}\right],
\end{aligned}
$$

where the second and fourth equality use that $|p|=|\tilde{p}|$ and $|q|=|\tilde{q}|$, and the third equality uses idempotency. Since $\eta$ recognises $\leq_{A}$ we have $i_{x}(u) \leq_{A} i_{x}(v)$ if, and only if, $i_{1}(u) \leq_{A} i_{1}(v)$. Due to the definition of $\leq_{Z}$, the latter is equivalent to $u \leq_{Z} v$. Finally, since $i_{x}$ is obviously a one-to-one map, this implies that $i_{x}$ is an embedding $i_{x}: Z \rightarrow B_{x}$.

Claim B.6. The map $f^{\prime}: \mathbb{N}^{n} \rightarrow \Sigma^{*}$ defined by

$$
f^{\prime}\left(x_{0}, \ldots, x_{n-1}\right)=\tilde{g}\left(x_{0}\right) h\left(x_{1}, \ldots, x_{n-1}\right)
$$

is an embedding $f^{\prime}: \mathbb{N}^{n} \hookrightarrow A$.

Proof. Consider $\bar{x}=\left(x_{0}, \ldots, x_{n-1}\right), \bar{y}=\left(y_{0}, \ldots, y_{n-1}\right) \in \mathbb{N}^{n}$ with $\bar{x}<\bar{y}$. Due to the definition of $f^{\prime}$ we have $f^{\prime}(\bar{x}) \in B_{x_{0}} \subseteq\left[g\left(x_{0}\right)\right]$. Analogously, $f^{\prime}(\bar{y}) \in\left[g\left(y_{0}\right)\right]$. We distinguish two cases. 
Case 1: $x_{0}<y_{0}$. Since $g$ is an embedding, we have $g\left(x_{0}\right)<g\left(y_{0}\right)$. That fact that $A=\sum_{w \in P}[w]$ implies $u<_{A} v$ for all $u \in\left[g\left(x_{0}\right)\right]$ and $v \in\left[g\left(y_{0}\right)\right]$. In particular, $f^{\prime}(\bar{x})<_{A} f^{\prime}(\bar{y})$.

Case 2: $x_{0}=y_{0}$. Let $\bar{x}^{\prime}=\left(x_{1}, \ldots, x_{n-1}\right), \bar{y}^{\prime}=\left(y_{1}, \ldots, y_{n-1}\right) \in \mathbb{N}^{n-1}$. Since $\bar{x}<\bar{y}$, we have $\bar{x}^{\prime}<\bar{y}^{\prime}$. For $h$ and $i_{x_{0}}=i_{y_{0}}$ are embeddings $h: \mathbb{N}^{n-1}$ this implies $h\left(\bar{x}^{\prime}\right)<_{Z} h\left(\bar{y}^{\prime}\right)$ and

$$
f^{\prime}(\bar{x})=i_{x_{0}}\left(h\left(\bar{x}^{\prime}\right)\right)<_{A} i_{y_{0}}\left(h\left(\bar{y}^{\prime}\right)\right)=f^{\prime}(\bar{y}) .
$$

Claim B.7. The map $f: \mathbb{N}^{n} \rightarrow A$ defined by

$$
f\left(x_{0}, \ldots, x_{n-1}\right)=f^{\prime}\left(K_{0} x_{0}+1, \ldots, K_{n-1} x_{n-1}+1\right)
$$

is a K-uniformly presentable.

Proof. For $i \in[0, n-1]$ factorise $v_{i}^{K_{i}}=p_{i} q_{i}$ such that

$$
\left|q_{i}\right|=\left|u_{0}\right|+\cdots+\left|u_{i}\right| \text {. }
$$

In addition, let $q_{-1}=\varepsilon$. For $i \in[0, n-1]$ put $\tilde{u}_{i}=q_{i-1} u_{i} p_{i}$ and $\tilde{v}_{i}=q_{i} p_{i}$. Further, let $\tilde{u}_{n}=q_{n-1} u_{n}$. For each $i \in[0, n-1]$ we obtain

$$
\left|\tilde{u}_{i}\right|=\left|q_{i-1}\right|+\left|u_{i}\right|+\left|p_{i}\right|=\left|u_{0}\right|+\cdots+\left|u_{i-1}\right|+\left|u_{i}\right|+\left|p_{i}\right|=\left|q_{i}\right|+\left|p_{i}\right|=K
$$

and

$$
\left|\tilde{v}_{i}\right|=\left|q_{i}\right|+\left|p_{i}\right|=K
$$

Moreover,

$$
\left|\tilde{u}_{n}\right|=\left|q_{n-1}\right|+\left|u_{n}\right|=\left|u_{0}\right|+\cdots+\left|u_{n-1}\right|+\left|u_{n}\right| \leq K
$$

For every $\bar{x}=\left(x_{0}, \ldots, x_{n-1}\right) \in \mathbb{N}^{n}$ we obtain

$$
\begin{aligned}
f\left(x_{0}, \ldots, x_{n-1}\right)= & f^{\prime}\left(K_{0} x_{0}+1, \ldots, K_{n-1} x_{n-1}+1\right) \\
= & u_{0} v_{0}^{K_{0} x_{0}} u_{1} v_{1}^{K_{1} x_{1}} \cdots u_{n-1} v_{n-1}^{K_{n-1} x_{n-1}} u_{n} \\
= & u_{0}\left(p_{0} q_{0}\right)^{x_{0}} u_{1}\left(p_{1} q_{1}\right)^{x_{1}} \cdots u_{n-1}\left(p_{n-1} q_{n-1}\right)^{x_{n-1}} u_{n} \\
= & u_{0} p_{0}\left(q_{0} p_{0}\right)^{x_{0}-1} q_{0} u_{1} p_{1}\left(q_{1} p_{1}\right)^{x_{1}-1} q_{1} \cdots \\
& \quad \cdot u_{n-1} p_{n-1}\left(q_{n-1} p_{n-1}\right)^{x_{n-1}-1} q_{n-1} u_{n} \\
= & \tilde{u}_{0} \tilde{v}_{0}^{x_{0}-1} \tilde{u}_{1} \tilde{v}_{1}^{x_{1}-1} \cdots \tilde{u}_{n-1} \tilde{v}_{n-1}^{x_{n-1}-1} \tilde{u}_{n} .
\end{aligned}
$$

This shows that $f$ is $K$-uniformly presentable.

This finishes the list of unproved non-trivial claims from the proof of Thm. 4.2. 


\section{B.2 Missing Details for Theorem 4.4}

Theorem 4.4. Let $n \geq 0$. The automatic Ramsey degree $d_{R, \mathrm{AUT}}\left(\omega^{n}\right)$ exists and is given by

$$
d_{R, \mathrm{AUT}}\left(\omega^{n}\right)=\sum_{i=1}^{n}\left\langle\begin{array}{c}
2 i-1 \\
i
\end{array}\right\rangle .
$$

The following fact was stated without evidence just before Thm. 4.4.

Lemma B.2. Let $n \geq 0$. There are precisely $\sum_{i=1}^{n}\left\langle\begin{array}{c}2 i-1 \\ i\end{array}\right\rangle$ restricted lower $n$ paths.

Proof. Using simultaneous induction on $i, j \in[0, n]$, we first show that there are precisely $\left\langle\begin{array}{c}i+j \\ j\end{array}\right\rangle$ paths through the 2-dimensional grid from $(n-i, n-j)$ to $(n, n)$ using only steps $(0,1),(1,0)$, and $(1,1)$, but the latter only on the main diagonal. For $i=j=0$ the claim is obvious. If $i=0$ and $j>0$, then there exists precisely one such path, namely $((n, n-j),(n, n-j+1), \ldots,(n, n))$. Since $\left\langle{ }_{j}^{j}\right\rangle=1$ this shows the claim. The case $i>0$ and $j=0$ is analogous. For $i, j>0$ there are two cases.

Case 1: $i \neq j$. Since the node $(n-i, n-j)$ is not on the main diagonal, we may not take a $(1,1)$-step. Thus we might either take a $(1,0)$-step and continue in from $(n-i+1, n-j)$ using one of the $\left\langle\begin{array}{c}i-1+j \\ j\end{array}\right\rangle$ paths allowed paths to $(n, n)$ or we take a $(0,1)$ and continue in one of $\left\langle\begin{array}{c}i+j-1 \\ j-1\end{array}\right\rangle$. Altogether, there are precisely $\left\langle\begin{array}{c}i-1+j \\ j\end{array}\right\rangle+\left\langle\begin{array}{c}i+j-1 \\ j-1\end{array}\right\rangle=\left\langle\begin{array}{c}i+j \\ j\end{array}\right\rangle$ allowed paths to $(n, n)$.

Case 2: $i=j$. First, we have the possibilities from Case 1. In addition, we may take a $(1,1)$-step since the node $(n-i, n-j)$ is on the main diagonal. There are $\left\langle\begin{array}{c}i-1+j-1 \\ i-1\end{array}\right\rangle$ allowed paths from $(n-i+1, n-j+1)$ to $(n, n)$. Altogether, there are $\left\langle\begin{array}{c}i-1+j \\ j\end{array}\right\rangle+\left\langle\begin{array}{c}i+j-1 \\ j-1\end{array}\right\rangle+\left\langle\begin{array}{c}i-1+j-1 \\ i-1\end{array}\right\rangle=\left\langle\begin{array}{c}i+j \\ j\end{array}\right\rangle$ allowed paths to $(n, n)$. This completes the induction.

Now, we consider restricted lower $n$-paths. Such an $n$-path first takes some number $j \in[0, n-1]$ of $(1,1)$-steps, then a (1,0)-step, and finally continues from $(j+1, j)$ to $(n, n)$. For a fixed $j$ there are $\left\langle\begin{array}{c}(n-j-1)+(n-j) \\ n-j\end{array}\right\rangle$ continuations. Thus, in total there are

$$
\sum_{j=0}^{n-1}\left\langle\begin{array}{c}
(n-j-1)+(n-j) \\
n-j
\end{array}\right\rangle=\sum_{i=1}^{n}\left\langle\begin{array}{c}
i-1+i \\
i
\end{array}\right\rangle
$$

$n$-paths.

The following non-trivial claims remained unproven in the proof of Thm. 4.2.

Claim B.8. The $\omega^{n}$-colouring $D:\left[\left\langle\mathbb{N}^{n}\right\rangle\right]^{2} \rightarrow Q$ with $D(\langle\bar{x}\rangle,\langle\bar{y}\rangle)=C(f(\bar{x}), f(\bar{y}))$ is automatic.

Proof. We have to show that for each $q \in Q$ the relation $D^{-1}(q) \subseteq\left\langle\mathbb{N}^{n}\right\rangle^{2}$ is automatic. Therefore, we fix some $q \in Q$ and proceed in three steps. 
Step 1. Consider the alphabet $\Gamma=[n] \cup\left\{\#_{i} \mid i \in[0, n]\right\}$, the one-to-one map $h: \mathbb{N}^{n} \rightarrow \Gamma^{*}$ defined by

$$
h\left(x_{0}, \ldots, x_{n-1}\right)=\#_{0} 0^{x_{0}-1} \#_{1} 1^{x_{1}-1} \cdots \#_{n-1}(n-1)^{x_{n-1}-1} \#_{n},
$$

and the relation

$$
R_{\#}=\left\{(h(\bar{x}), h(\bar{y})) \mid \bar{x}, \bar{y} \in \mathbb{N}^{n}, \bar{x} \neq \bar{y}, C(f(\bar{x}), f(\bar{y}))=q\right\} .
$$

In the rest of this step, we show that $R_{\#}$ is automatic.

Let $\left(u_{0}, v_{0}, \ldots, u_{n-1}, v_{n-1}, u_{n}\right)$ be the uniform presentation of $f$. We consider the unique morphism $\eta: \Gamma_{\diamond}^{*} \rightarrow \Sigma_{\diamond}^{*}$ with

$$
\eta(i)=v_{i}, \quad \eta\left(\#_{j}\right)=u_{j}, \quad \eta(\diamond)=\varepsilon
$$

for all $i \in[n]$ and $j \in[0, n]$. For $\bar{x} \in \mathbb{N}^{n}$ this construction yields

$$
f(\bar{x})=\eta(h(\bar{x})) .
$$

Let $\mu:\left(\Gamma_{\diamond}^{2}\right)^{*} \rightarrow\left(\Sigma_{\diamond}^{2}\right)^{*}$ be the unique morphism with

$$
\mu((\alpha, \beta))=\eta(\alpha) \otimes \eta(\beta)
$$

for all $(\alpha, \beta) \in \Gamma_{\diamond}^{2}$. Due to the uniformity of the presentation, we have for all $u, v \in \Gamma^{*}$ which contain $\#_{n}$ only as last letter that

$$
\eta(u) \otimes \eta(v)=\mu(u \otimes v) .
$$

Consequently, we obtain for $u, v \in \operatorname{im}(h)$ the following chain of equivalences:

$$
\begin{aligned}
(u, v) \in R_{\#} & \Longleftrightarrow(\eta(u), \eta(v)) \in C^{-1}(q) \\
& \Longleftrightarrow \mu(u \otimes v) \in \otimes C^{-1}(q) \\
& \Longleftrightarrow u \otimes v \in \mu^{-1}\left(\otimes C^{-1}(q)\right) .
\end{aligned}
$$

Put another way,

$$
\otimes R_{\#}=\mu^{-1}\left(\otimes C^{-1}(q)\right) \cap\left(\otimes\left(\operatorname{im}(h)^{2}\right) .\right.
$$

Therefore, $\otimes R_{\#}$ is regular, i.e., $R_{\#}$ is automatic.

Step 2. Consider the one-to-one correspondence

$$
G=\left\{(\langle\bar{x}\rangle, h(\bar{x})) \mid \bar{x} \in \mathbb{N}^{n}\right\} \subseteq[n]^{*} \times \Gamma^{*}
$$

between $\left\langle\mathbb{N}^{n}\right\rangle$ and $\operatorname{im}(h)$. For all $\bar{x}=\left(x_{0}, \ldots, x_{n-1}\right) \in \mathbb{N}^{n}$ we have

$$
\begin{aligned}
\langle\bar{x}\rangle \otimes h(\bar{x})=\left(0, \#_{0}\right)(0,0)^{x_{0}-1}\left(1, \#_{1}\right)(1,1)^{x_{1}-1} \cdots \\
\cdot\left(n-1, \#_{n-1}\right)(n-1, n-1)^{x_{n-1}-1}\left(\diamond, \#_{n}\right)
\end{aligned}
$$


Thus,

$$
\otimes G=\left(0, \#_{0}\right)(0,0)^{*}\left(1, \#_{1}\right)(1,1)^{*} \cdots\left(n-1, \#_{n-1}\right)(n-1, n-1)^{*}\left(\diamond, \#_{n}\right)
$$

is regular, i.e., $G$ is automatic.

For all $u, v \in\left\langle\mathbb{N}^{n}\right\rangle$ we have

$$
(u, v) \in D^{-1}(q) \quad \Longleftrightarrow \quad(G(u), G(v)) \in R_{\#} .
$$

Hence, the relation $D^{-1}(q)$ can be defined in the automatic structure $\left(\Gamma^{*} ; R_{\#}, G\right)$ by the formula

$$
\varphi_{D^{-1}(q)}(x, y)=\exists x^{\prime} \exists y^{\prime}\left(G\left(x, x^{\prime}\right) \wedge G\left(y, y^{\prime}\right) \wedge R_{\#}\left(x^{\prime}, y^{\prime}\right)\right) .
$$

Finally, $D^{-1}(q)$ is automatic by Thm. 2.1.

Claim B.9. For $\bar{x}, \bar{y} \in X_{M}$ the n-path $p(\bar{x}, \bar{y})$ is restricted.

Proof. Let $\bar{x}=\left(x_{0}, \ldots, x_{n-1}\right), \bar{y}=\left(y_{0}, \ldots, y_{n-1}\right) \in X_{m}$ and $\pi=p(\bar{x}, \bar{y})$. Assume that $\pi$ contains a $(1,1)$-step, say from $(i, j)$ to $(i+1, j+1)$. If $i+1=j+1=n$, this step is on the main diagonal. Now, additionally suppose that $i+1 \neq n$ or $j+1 \neq n$. Then $(i, j)(i+1, j+1)$ is a factor of $\langle\bar{x}\rangle \otimes\langle\bar{y}\rangle$, say $(i, j)$ is at position $k$. The $k^{\text {th }}$ letter of $\langle\bar{x}\rangle$ is an $i$, the last $i$ in $\langle\bar{x}\rangle$. Due to the specific choice of $X_{M}$ this implies $k \equiv(i+1) M(\bmod n M)$. Similarly, $k \equiv(j+1) M(\bmod n M)$. Thus, $i \equiv j(\bmod n)$. Since $0 \leq i, j<n$ we obtain $i=j$, i.e., the considered $(1,1)$-step is on the main diagonal.

Claim B.10. For $\bar{x}, \bar{y} \in X_{M}$ we have

$$
\eta(\langle\bar{x}\rangle \otimes\langle\bar{y}\rangle)=\eta\left(\sigma_{1}^{M} \cdots \sigma_{k}^{M}\right) .
$$

Proof. Write $\langle\bar{x}\rangle \otimes\langle\bar{y}\rangle=\sigma_{1}^{e_{1}} \cdots \sigma_{k}^{e_{k}}$ like in the definition of $p(\bar{x}, \bar{y})$. Since all the entries of $\bar{x}$ and $\bar{y}$ are divisible by $M$, each $e_{i}$ is divisible by $M$, say $e_{i}=d_{i} M$. Then

$$
\begin{aligned}
\eta(\langle\bar{x}\rangle \otimes\langle\bar{y}\rangle) & =\eta\left(\sigma_{1}^{d_{1} M} \cdots \sigma_{k}^{d_{k} M}\right) \\
& =\eta\left(\sigma_{1}\right)^{d_{i} M} \cdots \eta\left(\sigma_{k}\right)^{d_{k} M}=\eta\left(\sigma_{1}\right)^{M} \cdots \eta\left(\sigma_{k}\right)^{M}=\eta\left(\sigma_{1}^{M} \cdots \sigma_{k}^{M}\right),
\end{aligned}
$$

where the second equality uses idempotency.

Claim B.11. The set $f\left(X_{M}\right)$ is regular.

Proof. Let $\left(u_{0}, v_{0}, \ldots, u_{n-1}, v_{n-1}, u_{n}\right)$ be the uniform presentation of $f$. Then

$$
f\left(X_{M}\right)=u_{0} v_{0}^{M-1}\left(v_{0}^{n M}\right)^{*} u_{1} v_{1}^{M-1}\left(v_{1}^{n M}\right)^{*} \cdots u_{n-1} v_{n-1}^{M-1}\left(v_{n-1}^{n M}\right)^{*} u_{n}
$$

and this set is obviously regular.

Claim B.12. The $\omega^{n}$-colouring $C:\left[\left\langle X_{1}\right\rangle\right]^{2} \rightarrow Q$ with $C(\langle\bar{x}\rangle,\langle\bar{y}\rangle)=p(\bar{x}, \bar{y})$ for $\bar{x}<\bar{y}$ is automatic. 
Proof. We have to show that $C^{-1}(\pi)$ is automatic for every $\pi \in Q$. Therefore, we fix some $\pi=\left(\alpha_{1}, \beta_{1}\right) \ldots\left(\alpha_{k}, \beta_{k}\right)(n, n) \in Q$. Then we have

$$
\otimes\left(C^{-1}(\pi)\right)=\left(\alpha_{1}, \beta_{1}\right)^{+} \ldots\left(\alpha_{k}, \beta_{k}\right)^{+} \cup\left(\beta_{1}, \alpha_{1}\right)^{+} \ldots\left(\beta_{k}, \alpha_{k}\right)^{+},
$$

where the second set in the union contains all $(u, v) \in\left\langle X_{1}\right\rangle^{2}$ with $\left.u\right\rangle_{\operatorname{lex}} v$ and $C(u, v)=\pi$. Thus, $\otimes\left(C^{-1}(\pi)\right)$ is regular, i.e., $C^{-1}(\pi)$ is automatic.

Claim B.13. We have $C(f(n \cdot \bar{x}+\overline{1}), f(n \cdot \bar{y}+\overline{1}))=\pi$ for the unique $\bar{x}, \bar{y} \in \mathbb{N}^{n}$ with $\pi=(\langle\bar{x}\rangle \otimes\langle\bar{y}\rangle)(n, n)$.

Proof. For $n=0$ and $n=1$ the claim is trivially satisfied as there are no respectively just one restricted lower $n$-path. Thus, we may assume that $n \geq 2$. Let $\left(u_{0}, v_{0}, \ldots, u_{n-1}, v_{n-1}, u_{n}\right)$ be the uniform presentation of $f$, say it is $K$ uniform. From $f$ being a one-to-one map and $\operatorname{im}(f) \subseteq 0^{+} 1^{+} \ldots(n-1)^{+}$we conclude $v_{i}=i^{K}$ for $0 \leq i<n, u_{0} \in 0^{*}, u_{i} \in(i-1)^{*} i^{*}$ for $0<i<n$, and $u_{n} \in(n-1)^{*}$. Since $\operatorname{im}(f) \subseteq\left\langle X_{1}\right\rangle$, there is a unique $\bar{a} \in X_{1}$ such that $f(\overline{1})=\langle\bar{a}\rangle$. For every $\bar{z} \in \mathbb{N}^{n}$ we obtain $f(n \cdot \bar{z}+\overline{1})=\langle n K \cdot \bar{z}+\bar{a}\rangle$.

Since $\tilde{\pi}=C(f(n \cdot \bar{x}+\overline{1}), f(n \cdot \bar{y}+\overline{1}))$ and $\pi$ both are $n$-paths, it suffices to show that they contain the same points in the grid in order to prove that they are identical. First, assume that $(i, j)$ occurs at position $\ell$ in $\pi$. If $i=j=n$, then $(i, j)$ trivially also appears in $\pi$. There are three cases remaining, namely (1) $i, j<n,(2) i=n$ and $j<n$, and (3) $i<n$ and $j=n$. We only demonstrate case (1) as the other two cases can be treated similarly.

Now, assume $i, j<n$. The $\ell^{\text {th }}$ letter of $\langle\bar{x}\rangle$ is an $i$. The first $i$ in $\langle\bar{x}\rangle$ appears at position $x_{0}+\cdots+x_{i-1}+1$, the last one at position $x_{0}+\cdots+x_{i}$. Thus,

$$
x_{0}+\cdots+x_{i-1}+1 \leq \ell \leq x_{0}+\cdots+x_{i} .
$$

The first $i$ in $f(n \cdot \bar{x}+\overline{1})$ appears at position $n K \cdot\left(x_{0}+\cdots+x_{i-1}\right)+z_{0}+\cdots+z_{i-1}+1$, the last one at position $n K \cdot\left(x_{0}+\cdots+x_{i}\right)+z_{0}+\cdots+z_{i}$. Since $u_{n}$ does not contain one of the letters $0, \ldots, n-2$, we conclude

$$
z_{0}+\ldots+z_{n-2} \leq\left|u_{0}\right|+\ldots+\left|u_{n-1}\right| \leq n K .
$$

Since $z_{0}+\ldots+z_{n-2} \equiv n-1(\bmod n)$, we further conclude

$$
z_{0}+\ldots+z_{n-2} \leq n K-1 .
$$

Multiplying Eq. (9) and the inequalities above yields

$$
\begin{aligned}
n K \cdot\left(x_{0}+\cdots+x_{i-1}\right) & +z_{0}+\cdots+z_{i-1}+1 \\
& \leq n K \cdot\left(x_{0}+\cdots+x_{i-1}\right)+z_{0}+\cdots+z_{n-2}+1 \\
& \leq n K \cdot\left(x_{0}+\cdots+x_{i-1}+1\right) \\
& \leq n K \cdot \ell \\
& \leq n K \cdot\left(x_{0}+\cdots+x_{i}\right) \\
& \leq n K \cdot\left(x_{0}+\cdots+x_{i}\right)+z_{0}+\cdots+z_{i} .
\end{aligned}
$$


This means that the $(n K \cdot \ell)^{\text {th }}$ in $f(n \cdot \bar{x}+\overline{1})$ is $i$. Similarly, in $f(n \cdot \bar{y}+\overline{1})$ it is $j$ and in $f(n \cdot \bar{x}+\overline{1}) \otimes f(n \cdot \bar{y}+\overline{1})$ it is $(i, j)$. Thus, $\tilde{\pi}$ contains the letter $(i, j)$.

Finally, we have to show that $\tilde{\pi}$ contains no other symbols than those in $\pi$. The only way this could happen is that a $(1,1)$-step in $\pi$ is split into a $(1,0)$ and a $(0,1)$-step in $\tilde{\pi}$. However, we show that this actually cannot happen. Therefore, suppose there is a $(1,1)$-step from $(i, i)$ to $(i+1, i+1)$ with $i \in[n]$ in $\pi$. This implies that the positions of the last $i$ in $\langle\bar{x}\rangle$ and in $\langle\bar{y}\rangle$ coincide, i.e., $x_{0}+\cdots+x_{i}=y_{0}+\cdots+y_{i}$. We conclude that

$$
n K \cdot\left(x_{0}+\cdots+x_{i}\right)+z_{0}+\cdots+z_{i}=n K \cdot\left(y_{0}+\cdots+y_{i}\right)+z_{0}+\cdots+z_{i},
$$

i.e., the last positions of $i$ in $f(n \cdot \bar{x}+\overline{1})$ and in $f(n \cdot \bar{y}+\overline{1})$ also coincide. Hence, there also is a $(1,1)$-step from $(i, i)$ to $(i+1, i+1)$ in $\tilde{\pi}$.

This finishes the list of unproved non-trivial claims from the proof of Thm. 4.4.

\section{B.3 Missing Details for Theorem 4.7}

Theorem 4.7. Let $\alpha<\omega^{\omega}$ be an ordinal and $\alpha=\omega^{n_{1}}+\cdots+\omega^{n_{r}}$ with $r \geq 0$ and $n_{1} \geq \cdots \geq n_{r} \geq 0$ its Cantor normal form. The automatic Ramsey degree $d_{R, \mathrm{AUT}}(\alpha)$ exists and is given by

$$
d_{R, \mathrm{AUT}}(\alpha)=\sum_{i=1}^{r} d_{R, \mathrm{AUT}}\left(\omega^{n_{i}}\right)+\sum_{i=1}^{r} \sum_{j=i+1}^{r} d_{R, \mathrm{AUT}}\left(\omega^{n_{i}}, \omega^{n_{j}}\right) .
$$

Within the proof, we need the following.

Lemma B.3. For every ordinal $\alpha<\omega^{\omega}$ there exists a first-order sentence $\Phi_{\alpha}$ which is satisfied by a well-ordering if, and only if, it is of type $\alpha$.

Proof. Let $\alpha=\omega^{n_{1}}+\cdots+\omega^{n_{r}}$ with $r \geq 0$ and $n_{1} \geq \cdots \geq n_{r} \geq 0$ be the Cantor normal form of $\alpha$. We proceed by induction on $r$. If $r=0$ then $\alpha=0$ and we put

$$
\Phi_{0}=\neg \exists x(x=x) .
$$

The claim for $r=1$ was shown in Lemma B.1. Now, we assume $r \geq 2$. Let $\alpha^{\prime}=\omega^{n_{1}}+\cdots+\omega^{n_{r-1}}$ and put

$$
\Phi_{\alpha}=\exists x\left(\left.\Phi_{\alpha^{\prime}}\right|_{<x} \wedge \Phi_{\omega^{n_{r}} \Upsilon_{\geq x}}\right),
$$

where $\Phi_{\alpha^{\prime}}$ is relativised to all $y$ with $y<x$ and $\Phi_{\omega^{n_{r}}}$ to all $z$ with $z \geq x$.

Claim B.14. All the $A_{i}$ are effectively regular.

Proof. We fix an $i \in[i, r]$. Let $\alpha_{i}=\omega^{n_{1}}+\cdots+\omega^{n_{i-1}}$. The set $A_{i}$ is defined in $A$ by the formula

$$
\varphi_{A_{i}}(x)=\exists y\left(\left.\Phi_{\alpha_{i}}\right|_{<y} \wedge y \leq x \wedge \Phi_{<\omega^{n_{i}}} \uparrow_{[y, x)}\right),
$$

Hence, $A_{i}$ is effectively regular by Thm. 2.1. 
Claim B.15. We have

$$
|C(B)| \leq \sum_{i=1}^{r}\left|C\left(B_{i, r}\right)\right|+\sum_{i=1}^{r} \sum_{j=i+1}^{r}\left|C\left(B_{i, r} \times B_{j, r}\right)\right| \leq \mu(\alpha) .
$$

Proof. It holds that

$$
\begin{aligned}
C(B) & =\bigcup_{i=1}^{r} C\left(B_{i, r}\right) \cup \bigcup_{i=1}^{r} \bigcup_{j=i+1}^{r} C\left(B_{i, r} \times B_{j, r}\right) \\
& \subseteq \bigcup_{i=1}^{r} C\left(B_{i, 1}\right) \cup \bigcup_{i=1}^{r} \bigcup_{j=i+1}^{r} C\left(B_{i, j} \times B_{j, i+1}\right)
\end{aligned}
$$

and hence

$$
\begin{aligned}
|C(B)| & \leq \sum_{i=1}^{r}\left|C\left(B_{i, 1}\right)\right|+\sum_{i=1}^{r} \sum_{j=i+1}^{r}\left|C\left(B_{i, j} \times B_{j, i+1}\right)\right| \\
& \leq \sum_{i=1}^{r} d_{R, \mathrm{AUT}}\left(\omega^{n_{i}}\right)+\sum_{i=1}^{r} \sum_{j=i+1}^{r} d_{R, \mathrm{AUT}}\left(\omega^{n_{i}}, \omega^{n_{j}}\right) \\
& =\mu(\alpha)
\end{aligned}
$$

\section{Missing Proofs from Section 5}

The limit lemma characterises all $\Delta_{n}$ sets for $n \geq 2$ (sets computable in $\left.\emptyset^{(n-1)}\right)$. Here we only state the base case where $n=2$ as it is needed in the proof of Thm. 5.2. The reader is referred to [16, Chapter III] for details.

Lemma C.1 (Limit lemma). Any set $A \subseteq \mathbb{N}$ satisfies $A \in \Delta_{2}$ if and only if there is a computable function $f: \mathbb{N}^{2} \rightarrow\{0,1\}$ such that for any $x \in \mathbb{N}$, $(f(x, s))_{s \in \mathbb{N}}$ is eventually constant and $A=\{x \mid f(x, s)=1$ for almost all $s\}$.

Proposition 5.4. For every $k \in \mathbb{N}$, there exists a $k$-immune set partition $A_{1} \cup \cdots \cup A_{k}$ where each $A_{i}$ is a $\Delta_{2}$ set.

Proof. We list all $\Sigma_{1}$ sets as $W_{1}, W_{2}, \ldots$ and assume a uniformly effective finite approximation $\left(W_{e, s}\right)_{(e, s) \in \mathbb{N}}$. In other words $W_{e}=\bigcup_{s \in \mathbb{N}} W_{e, s}$ where each $W_{e, s} \subseteq$ $[1, s], W_{e, s} \subseteq W_{e, s+1}$ and $W_{e, s}(x)$ is computable given any $e, x \leq s \in \mathbb{N}$.

We describe the construction for the sets $A_{1}, \ldots, A_{k}$ that satisfy the following requirement for all $e \in \mathbb{N}$ :

$$
R_{e}: \quad\left|W_{e}\right|=\infty \Rightarrow\left(\bigwedge_{1 \leq i \leq k} W_{e} \cap A_{i} \neq \emptyset\right) .
$$


Note that satisfaction of all requirements $\left(R_{e}\right)_{e \in \mathbb{N}}$ will also guarantee that each of $A_{1}, \ldots, A_{k}$ is infinite: Indeed for each $y \in \mathbb{N}$ there is a $\Sigma_{1}$ set $W_{e}$ whose least element is greater than $y$. Thus $R_{e}$ ensures there is some elements $x>y$ that belongs to $A_{i}$ for $i \in[1, k]$.

The construction will define sets of numbers $\left(A_{i, s}\right)_{s \in \mathbb{N}}$ such that $\left(A_{1, s}, \ldots, A_{k, s}\right)$ is a partition of $\mathbb{N}$ for each $s$. The construction will also guarantee that $A_{i}=$ $\lim _{s \in \mathbb{N}} A_{i, s}(x)$ exists for each $x \in \mathbb{N}$. In other words $\forall x \in \mathbb{N} \exists t \forall s, s^{\prime} \geq t$ : $A_{i, s}(x)=A_{i, s^{\prime}}(x)=A_{i}(x)$. For any $x, s \in \mathbb{N}$ the value of $A_{i, s}(x)$ is computable and thus the set $A_{i}$ is $\Delta_{2}$ by the limit lemma.

To meet a single requirement $R_{e}$, we wait for a stage $s$ where $W_{e, s}$ contains at least $k$ elements $x_{1}, \ldots, x_{k}$. If no such stage $s$ exists, then $W_{e, s}$ is finite and $R_{e}$ is satisfied. Otherwise we act for $R_{e}$ by putting the element $x_{i}$ to the set $A_{i}$ where $1 \leq i \leq k$. We call the element $x_{i}$ the $i^{\text {th }}$ witness for $R_{e}$. If none of the witnesses $x_{1}, \ldots, x_{k}$ is taken out from their respective set at a later stage, the requirement $R_{e}$ is eventually met.

We order the requirements by decreasing priority as $R_{1}, R_{2}, R_{3}, \ldots$ When we want to meet a requirement $R_{e}$ using the witnesses $x_{1}, \ldots, x_{k}$, we may find that some $x_{i}$ has been used as a witness for another requirement $R_{e^{\prime}}$. If $R_{e^{\prime}}$ has higher priority than $R_{e}$, then $R_{e^{\prime}}$ forbids us to act for $R_{e}$ using $x_{1}, \ldots, x_{k}$. We use $M_{e, s}$ to denote the set of witnesses defined up to stage $s$ for requirements $R_{1}, \ldots, R_{e-1}$. We say a requirement $R_{e}$ requires attention at stage $s+1$ if $W_{e, s} \cap A_{i, s}=\emptyset$ for some $i \in[1, k]$ and $\left|W_{e, s} \backslash M_{e, s}\right| \geq k$.

Construction. At stage 0 , set $A_{1,0}=\mathbb{N}$ and $A_{i, 0}=\emptyset$ for each $i \in[2, k]$ and $M_{e, 0}=\emptyset$ for any $e \in \mathbb{N}$.

At stage $s>0$, if any $R_{e}$ requires attention, take the least such $e$. We act for $R_{e}$ as follows: Take the least $k$ elements $x_{1}<\ldots<x_{k}$ in $W_{e, s} \backslash M_{e, s}$. For each $i \in[1, k]$, assume $x_{i} \in A_{m_{i}, s-1}$ for some $m_{i} \in[1, k]$, we set $A_{i, s}=A_{i, s-1} \cup\left\{x_{i}\right\}$ and then take $x_{i}$ out of $A_{m_{i}, s}$ if $m_{i} \neq i$. We also set $M_{e^{\prime}, s}=M_{e^{\prime}, s-1} \cup\left\{x_{1}, \ldots, x_{k}\right\}$ for all $e^{\prime}>e$. If no $R_{e}$ requires attention, let $A_{i, s}=A_{i, s-1}$ and $M_{e, s}=M_{e, s-1}$ for each $i \in[1, k], e \in \mathbb{N}$. This finishes the construction at stage $s$. Note that $\left(A_{1, s}, \ldots, A_{k, s}\right)$ remains a partition of $\mathbb{N}$.

We verify the construction above using the following claims:

Claim. We act for each requirement $R_{e}$ only finitely many times.

Proof. By induction, let $s$ be the least stage after which no requirement $R_{e^{\prime}}$ with $e^{\prime}<e$ acts again. If $\left|W_{e} \backslash M_{e, s}\right|<k$ then $R_{e}$ will never act in future stages. Otherwise it will act once in a future stage $t>s$ when we define witnesses $x_{1}, \ldots, x_{k}$ for $R_{e}$ and these witnesses will never require attention again as $x_{1}, \ldots, x_{k}$ will belong to $M_{i, t^{\prime}}$ for any $i>e$ and $t^{\prime} \geq t$.

Claim. Suppose $\left|W_{e}\right|=\infty$. Then there is some $t \in \mathbb{N}$ and $x_{1}, \ldots, x_{k} \in W_{e}$ such that $\forall s \geq t: x_{i} \in A_{i, s}$ for $i \in[1, k]$.

Proof. By Claim C, let $s$ be the least stage after which no requirement $R_{e^{\prime}}$ with $e^{\prime}<e$ acts again. For any $t>s$ we have $M_{e, t}=M_{e, s}$. Since $W_{e}$ is infinite, 
there is some stage $t>s$ where $\left|W_{e, t} \backslash M_{e, t-1}\right| \geq k$. If $W_{e, t} \cap A_{i, t-1} \neq \emptyset$ for any $i \in[1, k]$. Then we would not act for $R_{e}$ and the statement in the claim is true. If $W_{e, t} \cap A_{i, t-1}=\emptyset$ for some $i \in[1, k]$, then we would act at stage $t$ for $R_{e}$ and the statement in the claim is true.

Claim $\mathrm{C}$ ensures that each requirement $R_{e}$ is met and by the discussion above the proposition is proved. 